\title{
高粱种子对萌发温度的响应分析与耐低温萌发能力鉴定
}

\author{
张瑞栋 ${ }^{1,2}$ \\ 肖梦颖 \\ 艾雪莹 1 \\ 周宇飞 1 ,* \\ 徐晓雪 ${ }^{1}$ \\ 姜 冰 ${ }^{1}$ \\ 邢艺凡 1 \\ 陈小飞 1 \\ 李 邦 ${ }^{1}$ \\ ${ }^{1}$ 沈阳农业大学农学院, 辽宁沈阳 $110866 ;{ }^{2}$ 山西省农业科学院经济作物研究所, 山西汾阳 032200
}

摘 要: 萌发期的低温是限制种子萌发的一个重要非生物胁迫因子, 高粱种子耐低温萌发能力是保证高粱出苗整 齐、建立良好群体的基础, 因此研究高粱品种对不同萌发温度的响应特征具有重要的理论和应用价值。本试验用来 源于不同地区的 30 份高粱品种, 在 $25^{\circ} \mathrm{C} 、 20^{\circ} \mathrm{C} 、 16^{\circ} \mathrm{C}$ 和 $12^{\circ} \mathrm{C}$ 人工气候箱中进行萌发试验, 测定不同温度下高粱品种 的发芽势、发芽率、芽长、根长、芽重、根重等萌发指标，分析不同高粱品种的萌发差异; 通过主成分分析和聚类分 析, 对不同高粱品种萌发期耐低温特性鉴定和分类, 结果表明, 随着萌发温度的降低, 高粱芽和根的生长均受到抑 制, 但根与芽的重量和长度比均增加, 说明萌发过程中芽比根对低温更加敏感。30 个高粱品种的 6 个萌发指标相对 值之间存在一定相关性。主成分分析表明, 相对芽长、相对根长和相对发芽率分别在 3 个主成分中载荷较大, 可作为 高粱萌发期耐低温的主要鉴定指标。30个高粱品种按萌发期耐低温能力可分为四大类, 辽粘 3 号极不耐低温, 济粱 1 号等 18 个品种对低温敏感, 冀酿 1 号等 5 个品种对低温不敏感, 赤杂 101 等 6 个品种对萌发期低温具有较强的耐性。 关键词: 高粱; 萌发特征; 低温敏感性; 主成分分析

\section{Responses of sorghum hybrids to germination temperatures and identification of low temperature resistance}

ZHANG Rui-Dong $^{1,2}$, XIAO Meng-Ying ${ }^{1}$, XU Xiao-Xue ${ }^{1}$, JIANG Bing, XING Yi-Fan ${ }^{1}$, CHEN Xiao-Fei $^{1}$, LI Bang $^{1}$, AI Xue-Ying ${ }^{1}$, ZHOU Yu-Fei ${ }^{1, *}$, and HUANG Rui-Dong

${ }^{1}$ College of Agronomy, Shenyang Agricultural University, Shenyang 110866, Liaoning, China; ${ }^{2}$ Institute of Cash Crops, Shanxi Academy of Agricultural Sciences, Fenyang 032200, Shanxi, China

\begin{abstract}
Low temperature during germination is an important abiotic stress factor limiting seed germination. Sorghum seeds with high germination capacity at low temperature are conducive to the emergence and establishment of a good population. Therefore, studying the response characteristics of sorghum hybrids to different germination temperatures is of great theoretical and practical value. Thirty sorghum hybrids from different regions of China were germinated in the artificial climate chamber, at $25^{\circ} \mathrm{C}, 20^{\circ} \mathrm{C}, 16^{\circ} \mathrm{C}$, or $12^{\circ} \mathrm{C}$ to measure germination potential, germination percent, bud length, root length, bud weight and root weight. Sorghum hybrids were identified and classified according to low temperature resistance during the germination based on principal component analysis (PCA) and clustering analysis. With a decrease in germination temperature, the growth of both buds and roots were inhibited, but the ratio of roots to buds in both weight and length increased, indicating that buds were more sensitive to low temperature during germination than roots. The relative values of six germination indicators of the thirty sorghum hybrids were correlated between each other. The PCA results showed that relative bud length, relative root length and relative germination percent could be used to evaluate the sorghum germination capacity under low temperatures because these indicators had the largest loads in the three principal components, respectively. According to the low temperature tolerance ability of the 30 sorghum hybrids were divided into four categories: Liaonian 3 was extremely sensitive to low temperature; eighteen hybrids, such as Jiliang 1, were sensitive to low temperature; five hybrids, such as Jiniang 1, were insensitive to low temperature, and six hybrids, such as Chiza 101 had a strong low temperature tolerance during the germination.
\end{abstract}

\section{本研究由国家现代农业产业技术体系建设专项(CARS-06-135-A17)资助。}

This study was supported by the Earmarked Fund for the China Agriculture Research System (CARS-06-135-A17).

* 通信作者(Corresponding author): 周宇飞, E-mail: zhouyufei2002@aliyun.com; zhouyufei@syau.edu.cn

第一作者联系方式: E-mail: sxnkyzrd@126.com

Received (收稿日期): 2019-10-08; Accepted (接受日期): 2020-01-15; Published online (网络出版日期): 2020-01-23.

URL: http://kns.cnki.net/kcms/detail/11.1809.S.20200123.1103.002.html 
Keywords: sorghum; germination traits; low temperature sensitivity; principal components analysis

高粱是我国北方地区重要的粮食作物, 具有多 重抗性 ${ }^{[1]}$ 。然而, 高粱对低温比较敏感, 而且不同生 育阶段对低温的敏感性不同，萌发期是高粱对低温 最为敏感的时期 ${ }^{[2]} 。 0 \sim 20^{\circ} \mathrm{C}$ 之间的温度都会对高粱 的萌发和生长造成不同程度的影响 ${ }^{[3]}$ 。春季低温常 常会导致高粱种子粉种、霉烂, 出苗困难, 进而影响 产量 ${ }^{[4]}$ 。筛选具有较高耐低温萌发能力的高粱品种, 并鉴选其主要评价指标, 对降低高粱早春播种的风 险和拓展高粱的种植区域具有重要的理论和实践意 $义^{[5]}$ 。种子萌发是一个非常复杂的生物过程, 包括胚 乳和种皮的破裂, DNA、细胞膜和细胞器的修复, 核 酸和蛋白的合成, 糖和蛋白的代谢等 ${ }^{[6-9]}$ 。温度是影 响酶活性和细胞生命活动的一个关键因子 ${ }^{[10]}$, 萌发 期的低温会改变种子内部的生理活性, 进而影响种 子的萌发能力 ${ }^{[11]}$ 。Upadhyaya 等 ${ }^{[12]}$ 发现 $12^{\circ} \mathrm{C}$ 显著影 响高粱种子的发芽能力, 可作为高粱萌发期的鉴定 温度。Franks 等 ${ }^{[13]}$ 研究认为 $15^{\circ} \mathrm{C}$ 适合作为高粱萌发 期耐低温的篮选温度。张丽霞等 ${ }^{[14]}$ 认为 $8^{\circ} \mathrm{C}$ 下的相 对萌发率和相对萌发势可评价高粱的耐低温萌发能 力。张海燕等 ${ }^{[15]}$ 认为可以通过 $5 \mathrm{~d} 2^{\circ} \mathrm{C}$ 的低温处理, 然后转移到 $12 \sim 15^{\circ} \mathrm{C}$ 培养, 根据出苗情况篮选高粱 的耐低温种质资源。可见前人在评价高粱萌发期耐 低温条件上存在许多差异, 而指标的选取对作物耐 低温萌发能力的评价同样具有重要的影响。朱晨曦 等 ${ }^{[16]}$ 研究发现, $18^{\circ} \mathrm{C}$ 培养下的相对发芽指数或者 $14^{\circ} \mathrm{C}$ 培养下的相对发芽率可作为辣椒种子萌发耐低 温的鉴定指标。王俊娟等 ${ }^{[17]}$ 研究认为 $0^{\circ} \mathrm{C}$ 处理 $4 \mathrm{~d}$, $28^{\circ} \mathrm{C}$ 条件下恢复正常生长 $7 \mathrm{~d}$ 的相对子叶平展率可 以作为棉花萌发期的抗冷鉴定指标。单一的萌发指 标或者植株形态指标在评价作物某一方面的耐低温 性上具有一定的参考价值, 但不能反映出品种耐低 温萌发的综合能力。张雪峰等 ${ }^{[18]}$ 和高利英等 ${ }^{[19]}$ 分别 通过发芽率、发芽势、发芽指数和活力指数的综合 变化评价了玉米和棉花萌发期的耐低温特性, 但是 这些指标之间存在一定的关联, 指标所反映的信息 有一定的重叠, 指标的选取对鉴定结果有一定的影 响。高粱品种萌发过程中对不同低温敏感性存在差 异, 多性状指标的评价可以综合反映高粱的耐低温 能力。然而, 到目前为止, 萌发期高粱品种耐低温能 力的综合评价还鲜见报道。本试验以 30 份高粱品种 为研究材料, 通过对不同萌发温度条件下的多个萌 发性状进行分析，旨在篮选出高粱萌发期耐低温的 主要鉴定指标和萌发期耐低温的高粱品种。

\section{1 材料与方法}

\section{1 供试材料}

选用中国不同生态区的 30 个高粱品种, 均为所 在区域推广使用的最新品种, 分别来自山东省农业 科学院、辽宁省农业科学院、吉林省农业科学院、 四川省农业科学院、山西省农业科学院、河北省农 林科学院、锦州市科学技术研究院(辽宁省)、通辽市 农业科学研究院(内蒙古自治区)、平凉市农业科学院 (甘肃省)、赤峰市农牧科学院(内蒙古自治区)等单 位。品种名称及来源见表 1 。

\section{表 1 供试高粱品种及来源}

Table 1 Tested sorghum hybrids and their origin

\begin{tabular}{|c|c|c|}
\hline 编号 & 名称 & 品种来源 \\
\hline Serial number & Cultivar & Origin \\
\hline S1 & 济粱 1 号 Jiliang 1 & 山东省农业科学院 Shandong Academy of Agricultural Sciences \\
\hline S2 & 济粱 2 号 Jiliang 2 & 山东省农业科学院 Shandong Academy of Agricultural Sciences \\
\hline S3 & 冀酿 1 号 Jiniang 1 & 河北省农林科学院 Hebei Academy of Agricultural and Forestry Sciences \\
\hline S4 & 冀酿 2 号 Jiniang 2 & 河北省农林科学院 Hebei Academy of Agricultural and Forestry Sciences \\
\hline S5 & 辽粘 3 号 Liaonian 3 & 辽宁省农业科学院 Liaoning Academy of Agricultural Sciences \\
\hline S6 & 辽糯 11 Liaonuo 11 & 辽宁省农业科学院 Liaoning Academy of Agricultural Sciences \\
\hline S7 & 辽杂 19 Liaoza 19 & 辽宁省农业科学院 Liaoning Academy of Agricultural Sciences \\
\hline S8 & 辽杂 27 Liaoza 27 & 辽宁省农业科学院 Liaoning Academy of Agricultural Sciences \\
\hline S9 & 辽杂 36 Liaoza 36 & 辽宁省农业科学院 Liaoning Academy of Agricultural Sciences \\
\hline $\mathrm{S} 10$ & 辽杂 37 Liaoza 37 & 辽宁省农业科学院 Liaoning Academy of Agricultural Sciences \\
\hline S11 & $13163 \mathrm{~A} /$ 早 $21 \mathrm{R} 13163 \mathrm{~A} / \mathrm{Zao} 21 \mathrm{R}$ & 四川省农业科学院 Sichuan Academy of Agricultural Sciences \\
\hline
\end{tabular}




\begin{tabular}{|c|c|c|}
\hline $\begin{array}{c}\text { 编号 } \\
\text { Serial number }\end{array}$ & $\begin{array}{c}\text { 名称 } \\
\text { Cultivar }\end{array}$ & $\begin{array}{c}\text { 品种来源 } \\
\text { Origin } \\
\end{array}$ \\
\hline S12 & 3401A/早 21R 3401A/Zao 21R & 四川省农业科学院 Sichuan Academy of Agricultural Sciences \\
\hline S13 & 吉杂 127 Jiza 127 & 吉林省农业科学院 Jilin Academy of Agricultural Sciences \\
\hline S14 & 吉杂 136 Jiza 136 & 吉林省农业科学院 Jilin Academy of Agricultural Sciences \\
\hline S15 & 吉杂 137 Jiza 137 & 吉林省农业科学院 Jilin Academy of Agricultural Sciences \\
\hline S16 & 吉杂 145 Jiza 145 & 吉林省农业科学院 Jilin Academy of Agricultural Sciences \\
\hline S17 & 吉杂 152 Jiza 152 & 吉林省农业科学院 Jilin Academy of Agricultural Sciences \\
\hline S18 & 吉杂 305 Jiza 305 & 吉林省农业科学院 Jilin Academy of Agricultural Sciences \\
\hline S19 & 通杂 108 Tongza 108 & 通辽市农业科学研究院 Tongliao Academy of Agricultural Sciences \\
\hline S20 & 通杂 137 Tongza 137 & 通辽市农业科学研究院 Tongliao Academy of Agricultural Sciences \\
\hline S21 & $407 / 272$ & 四川省农业科学院 Sichuan Academy of Agricultural Sciences \\
\hline S22 & 407/M5 & 四川省农业科学院 Sichuan Academy of Agricultural Sciences \\
\hline S23 & 锦杂 107 Jinza 107 & 锦州市科学技术研究院 Jinzhou Academy of Science and Technology \\
\hline S24 & 锦杂 109 Jinza 109 & 锦州市科学技术研究院 Jinzhou Academy of Science and Technology \\
\hline S25 & 晋中 0592 Jinzhong 0592 & 山西省农业科学院 Shanxi Academy of Agricultural Sciences \\
\hline S26 & 晋中 0742 Jinzhong 0742 & 山西省农业科学院 Shanxi Academy of Agricultural Sciences \\
\hline S27 & 晋杂 31 Jinza 31 & 山西省农业科学院 Shanxi Academy of Agricultural Sciences \\
\hline S28 & 晋杂 33 Jinza 33 & 山西省农业科学院 Shanxi Academy of Agricultural Sciences \\
\hline S29 & 平试 13 Pingshi 13 & 平凉市农业科学院 Pingliang Academy of Agricultural Sciences \\
\hline $\mathrm{S} 30$ & 赤杂 101 Chiza 101 & 赤峰市农牧科学院 Chifeng Academy of Agricultural and Animal Husbandry Sciences \\
\hline
\end{tabular}

\section{2 试验设计}

采用 RXZ-1000B型人工气候箱进行种子控温萌 发培养, 设湿度为 $60 \%$, 黑暗培养 $10 \mathrm{~d}$ 。挑选籽粒饱 满的种子, 用次氯酸钠溶液 $(5 \%)$ 消毒 $15 \mathrm{~min}$, 用纯 水冲净拭干，置铺好滤纸的培养血中，每个培养血 放 25 粒, 作好标记, 加入 $15 \mathrm{~mL}$ 蒸馏水, 摆放好种 子后置人工气候箱。从培养的第 3 天开始, 统计发 芽势，每 $2 \mathrm{~d}$ 补充一次蒸馏水。在正式试验之前以济 粱 1 号、辽杂 37 、赤杂 101 以及辽粘 3 号进行预备 发芽试验, 基于 $10^{\circ} \mathrm{C}$ 为喜温作物的界限温度, 选取 $25^{\circ} \mathrm{C} 、 20^{\circ} \mathrm{C} 、 16^{\circ} \mathrm{C} 、 12^{\circ} \mathrm{C}$ 和 $8^{\circ} \mathrm{C}$ 为发芽温度。结果发 现这些高粱品种在 $8^{\circ} \mathrm{C}$ 下培养 $10 \mathrm{~d}$ 均不发芽, 故舍 弃 $8^{\circ} \mathrm{C}$ 而选取 $25^{\circ} \mathrm{C} 、 20^{\circ} \mathrm{C} 、 16^{\circ} \mathrm{C}$ 和 $12^{\circ} \mathrm{C} 4$ 个发芽温 度进行发芽试验。

\section{3 测定指标}

在第 10 天时从每个培养血随机取 5 个样品, 测 定芽长、根长、芽鲜重、根鲜重等指标, 取其平均值。

发芽以种子胚根达种子长, 胚芽达种子长的二 分之一为标准。

选取各处理下长势一致的高粱幼苗, 从培养血 中完整地取出植株, 拭干水分后用直尺测量芽长(芽 基部到上部最长叶的绝对距离)、根长(植株基部到根
系形态学最下端的绝对距离)。

将洗净、拭干后的植株在根茎结合处剪断, 用 电子天平分别称量芽和根的鲜重。

发芽势 $=$ 第 3 天的发芽种子数/供试种子数 $\times 100 \%$

发芽率 = 第 10 天的发芽种子数/供试种子数 $\times 100 \%$

萌发指标相对值 $(\%)=\sum\left(\frac{T_{\mathrm{CK}}-T_{\mathrm{t}}}{T_{\mathrm{CK}}-T_{\mathrm{t}}} \times \frac{V_{\mathrm{t}}}{V_{\mathrm{CK}}}\right) \times 100$ 其中, $T_{\mathrm{t}} 、 T_{\mathrm{ck}} 、 V_{\mathrm{t}}$ 和 $V_{\mathrm{CK}}$ 分别为处理温度、对照温度、 处理指标值和对照指标值。

\section{4 数据处理与分析}

用 Microsoft Excel 2007 整理数据并计算各处理 性状的平均值和相对值。用 SPSS 18.0 进行方差分 析、相关分析和主成分分析，通过载荷值篮选鉴定 指标，依据主成分的综合得分对高粱耐低温特性进 行聚类评价。

\section{2 结果与分析}

2.1 不同高粱品种萌发指标对温度的响应

由表 2 可知, 在不同品种之间, 发芽势、发芽 率、芽长、根长、芽重和根重的差异达到极显著水 
表 2 不同温度下高粱品种萌发指标的差异比较

Table 2 Comparison of germination indicators of sorghum under different temperatures

\begin{tabular}{|c|c|c|c|c|c|c|c|}
\hline $\begin{array}{c}\text { 品种 } \\
\text { Hybrid }\end{array}$ & $\begin{array}{c}\text { 温度 } \\
\text { Temperature } \\
\left({ }^{\circ} \mathrm{C}\right)\end{array}$ & $\begin{array}{c}\text { 发芽势 } \\
\text { Germination } \\
\text { potential (\%) }\end{array}$ & $\begin{array}{c}\text { 发芽率 } \\
\text { Germination } \\
\text { percent (\%) }\end{array}$ & $\begin{array}{c}\text { 芽长 } \\
\text { Bud length } \\
(\mathrm{cm}) \\
\end{array}$ & $\begin{array}{c}\text { 根长 } \\
\text { Root length } \\
(\mathrm{cm}) \\
\end{array}$ & $\begin{array}{c}\text { 芽重 } \\
\text { Bud FW } \\
\text { (mg) }\end{array}$ & $\begin{array}{c}\text { 根重 } \\
\text { Root FW } \\
(\mathrm{mg})\end{array}$ \\
\hline \multirow[t]{4}{*}{ S1 } & 25 & $78.67 \pm 6.11$ & $84.90 \pm 4.29$ & $9.90 \pm 0.93$ & $13.17 \pm 2.38$ & $92.00 \pm 7.77$ & $72.17 \pm 7.96$ \\
\hline & 20 & $73.33 \pm 6.11$ & $84.67 \pm 2.31$ & $4.92 \pm 0.62$ & $12.80 \pm 1.65$ & $54.50 \pm 10.48$ & $76.17 \pm 11.89$ \\
\hline & 16 & $33.67 \pm 4.51$ & $82.33 \pm 6.03$ & $3.35 \pm 0.31$ & $6.60 \pm 0.88$ & $25.00 \pm 3.10$ & $27.50 \pm 3.02$ \\
\hline & 12 & $0.00 \pm 0.00$ & $74.67 \pm 12.86$ & $0.32 \pm 0.04$ & $1.19 \pm 0.17$ & $1.83 \pm 0.26$ & $8.17 \pm 0.75$ \\
\hline \multirow[t]{4}{*}{ S2 } & 25 & $81.33 \pm 2.31$ & $84.67 \pm 2.52$ & $10.37 \pm 1.51$ & $9.00 \pm 1.46$ & $89.67 \pm 13.17$ & $25.00 \pm 4.94$ \\
\hline & 20 & $80.57 \pm 3.44$ & $84.33 \pm 4.04$ & $6.02 \pm 0.90$ & $15.42 \pm 2.80$ & $62.50 \pm 6.12$ & $64.03 \pm 12.69$ \\
\hline & 16 & $63.33 \pm 3.06$ & $80.00 \pm 2.00$ & $2.02 \pm 0.16$ & $7.52 \pm 0.72$ & $20.60 \pm 3.83$ & $63.20 \pm 9.45$ \\
\hline & 12 & $0.00 \pm 0.00$ & $72.00 \pm 4.00$ & $0.42 \pm 0.04$ & $1.45 \pm 0.14$ & $2.50 \pm 0.46$ & $11.50 \pm 1.52$ \\
\hline \multirow[t]{4}{*}{ S3 } & 25 & $68.00 \pm 4.00$ & $89.33 \pm 2.31$ & $9.03 \pm 0.65$ & $13.60 \pm 2.05$ & $75.00 \pm 8.29$ & $74.33 \pm 14.62$ \\
\hline & 20 & $65.33 \pm 7.02$ & $88.00 \pm 3.46$ & $5.77 \pm 0.64$ & $12.52 \pm 1.42$ & $57.17 \pm 5.91$ & $65.67 \pm 9.58$ \\
\hline & 16 & $21.33 \pm 1.53$ & $87.33 \pm 3.06$ & $1.98 \pm 0.21$ & $6.85 \pm 0.90$ & $22.00 \pm 2.90$ & $37.50 \pm 5.99$ \\
\hline & 12 & $0.00 \pm 0.00$ & $84.00 \pm 6.93$ & $0.37 \pm 0.08$ & $1.40 \pm 0.13$ & $2.33 \pm 0.29$ & $10.67 \pm 1.21$ \\
\hline \multirow[t]{4}{*}{ S4 } & 25 & $60.00 \pm 4.00$ & $78.33 \pm 6.03$ & $9.47 \pm 1.16$ & $6.62 \pm 0.66$ & $70.67 \pm 8.82$ & $17.17 \pm 1.47$ \\
\hline & 20 & $58.67 \pm 2.31$ & $76.00 \pm 5.29$ & $5.18 \pm 0.73$ & $10.18 \pm 0.83$ & $55.17 \pm 4.58$ & $57.67 \pm 9.58$ \\
\hline & 16 & $37.33 \pm 2.31$ & $68.23 \pm 3.28$ & $1.86 \pm 0.14$ & $4.92 \pm 0.68$ & $21.80 \pm 4.23$ & $44.80 \pm 8.86$ \\
\hline & 12 & $0.00 \pm 0.00$ & $38.67 \pm 5.03$ & $0.40 \pm 0.06$ & $1.08 \pm 0.12$ & $1.50 \pm 0.41$ & $6.50 \pm 0.55$ \\
\hline \multirow[t]{4}{*}{ S5 } & 25 & $72.00 \pm 4.00$ & $74.00 \pm 4.00$ & $11.53 \pm 1.20$ & $14.47 \pm 1.98$ & $106.17 \pm 15.78$ & $64.67 \pm 7.17$ \\
\hline & 20 & $31.33 \pm 1.53$ & $66.67 \pm 2.31$ & $4.40 \pm 0.46$ & $7.83 \pm 1.16$ & $49.50 \pm 5.24$ & $40.00 \pm 6.63$ \\
\hline & 16 & $0.00 \pm 0.00$ & $61.33 \pm 8.33$ & $2.10 \pm 0.29$ & $4.62 \pm 0.45$ & $24.00 \pm 4.15$ & $26.33 \pm 3.27$ \\
\hline & 12 & $0.00 \pm 0.00$ & $10.67 \pm 4.62$ & $0.10 \pm 0.01$ & $0.72 \pm 0.13$ & $0.48 \pm 0.15$ & $3.33 \pm 0.52$ \\
\hline \multirow[t]{4}{*}{ S6 } & 25 & $93.33 \pm 4.62$ & $94.67 \pm 2.31$ & $10.97 \pm 0.96$ & $14.80 \pm 2.23$ & $102.00 \pm 14.64$ & $59.33 \pm 11.45$ \\
\hline & 20 & $84.00 \pm 5.29$ & $93.67 \pm 2.08$ & $5.10 \pm 0.28$ & $10.58 \pm 1.66$ & $64.83 \pm 8.47$ & $56.00 \pm 4.43$ \\
\hline & 16 & $22.67 \pm 2.31$ & $93.33 \pm 4.62$ & $2.47 \pm 0.16$ & $5.67 \pm 0.75$ & $28.67 \pm 4.18$ & $27.83 \pm 4.22$ \\
\hline & 12 & $0.00 \pm 0.00$ & $81.33 \pm 4.62$ & $0.27 \pm 0.05$ & $1.27 \pm 0.16$ & $2.00 \pm 0.52$ & $8.33 \pm 1.03$ \\
\hline \multirow[t]{4}{*}{ S7 } & 25 & $86.00 \pm 4.00$ & $95.33 \pm 1.15$ & $11.80 \pm 0.98$ & $9.83 \pm 1.05$ & $108.58 \pm 14.56$ & $19.17 \pm 3.13$ \\
\hline & 20 & $81.33 \pm 8.33$ & $94.67 \pm 1.53$ & $6.68 \pm 0.26$ & $11.73 \pm 2.10$ & $72.67 \pm 10.89$ & $52.50 \pm 7.82$ \\
\hline & 16 & $80.00 \pm 4.00$ & $94.33 \pm 3.21$ & $2.28 \pm 0.21$ & $6.72 \pm 1.12$ & $22.20 \pm 2.79$ & $27.40 \pm 5.00$ \\
\hline & 12 & $0.00 \pm 0.00$ & $88.00 \pm 4.00$ & $0.63 \pm 0.12$ & $2.40 \pm 0.42$ & $4.17 \pm 0.75$ & $12.00 \pm 0.89$ \\
\hline \multirow[t]{4}{*}{ S8 } & 25 & $97.33 \pm 2.31$ & $98.67 \pm 2.31$ & $11.13 \pm 1.41$ & $8.23 \pm 1.37$ & $77.50 \pm 15.14$ & $21.17 \pm 3.37$ \\
\hline & 20 & $92.23 \pm 5.08$ & $95.67 \pm 3.21$ & $5.77 \pm 0.71$ & $13.78 \pm 1.50$ & $46.50 \pm 5.68$ & $55.83 \pm 7.65$ \\
\hline & 16 & $82.00 \pm 3.46$ & $92.67 \pm 3.06$ & $1.92 \pm 0.07$ & $6.26 \pm 0.66$ & $15.20 \pm 1.60$ & $31.40 \pm 3.72$ \\
\hline & 12 & $0.00 \pm 0.00$ & $90.67 \pm 2.31$ & $0.40 \pm 0.06$ & $1.65 \pm 0.10$ & $1.17 \pm 0.41$ & $7.00 \pm 0.89$ \\
\hline \multirow[t]{4}{*}{ S9 } & 25 & $84.43 \pm 5.10$ & $93.33 \pm 2.31$ & $9.10 \pm 0.94$ & $13.65 \pm 1.96$ & $99.00 \pm 14.76$ & $38.67 \pm 4.59$ \\
\hline & 20 & $68.90 \pm 6.97$ & $93.33 \pm 2.31$ & $8.70 \pm 1.28$ & $15.02 \pm 1.99$ & $75.67 \pm 6.98$ & $64.83 \pm 5.27$ \\
\hline & 16 & $18.67 \pm 1.53$ & $92.00 \pm 4.00$ & $2.40 \pm 0.20$ & $6.35 \pm 0.86$ & $32.17 \pm 4.92$ & $40.83 \pm 6.74$ \\
\hline & 12 & $0.00 \pm 0.00$ & $90.00 \pm 2.00$ & $0.67 \pm 0.08$ & $1.93 \pm 0.23$ & $7.83 \pm 1.33$ & $14.33 \pm 2.42$ \\
\hline \multirow[t]{4}{*}{$\mathrm{S} 10$} & 25 & $73.33 \pm 5.03$ & $90.67 \pm 2.31$ & $11.45 \pm 0.98$ & $13.88 \pm 2.72$ & $85.33 \pm 10.19$ & $62.67 \pm 3.61$ \\
\hline & 20 & $53.33 \pm 3.06$ & $89.33 \pm 2.31$ & $5.72 \pm 0.74$ & $11.58 \pm 2.17$ & $52.33 \pm 6.56$ & $56.17 \pm 8.35$ \\
\hline & 16 & $33.33 \pm 4.16$ & $86.67 \pm 4.62$ & $2.65 \pm 0.26$ & $6.98 \pm 1.05$ & $27.17 \pm 4.75$ & $28.83 \pm 0.75$ \\
\hline & 12 & $0.00 \pm 0.00$ & $58.67 \pm 8.33$ & $0.17 \pm 0.05$ & $1.27 \pm 0.23$ & $1.33 \pm 0.39$ & $7.00 \pm 1.10$ \\
\hline \multirow[t]{3}{*}{$\mathrm{S} 11$} & 25 & $61.33 \pm 6.11$ & $85.33 \pm 2.31$ & $8.92 \pm 0.77$ & $7.70 \pm 1.00$ & $81.83 \pm 6.21$ & $33.33 \pm 4.32$ \\
\hline & 20 & $58.00 \pm 6.00$ & $82.67 \pm 2.31$ & $5.60 \pm 0.49$ & $10.67 \pm 1.35$ & $54.50 \pm 6.66$ & $61.50 \pm 10.56$ \\
\hline & 16 & $12.33 \pm 1.53$ & $80.00 \pm 4.00$ & $2.45 \pm 0.19$ & $5.30 \pm 0.83$ & $24.67 \pm 3.67$ & $30.17 \pm 3.60$ \\
\hline
\end{tabular}


(续表 2)

\begin{tabular}{|c|c|c|c|c|c|c|c|}
\hline $\begin{array}{c}\text { 品种 } \\
\text { Hybrid }\end{array}$ & $\begin{array}{c}\text { 温度 } \\
\text { Temperature } \\
\left({ }^{\circ} \mathrm{C}\right) \\
\end{array}$ & $\begin{array}{c}\text { 发芽势 } \\
\text { Germination } \\
\text { potential (\%) }\end{array}$ & $\begin{array}{c}\text { 发芽率 } \\
\text { Germination } \\
\text { percent }(\%)\end{array}$ & $\begin{array}{c}\text { 芽长 } \\
\text { Bud length } \\
(\mathrm{cm}) \\
\end{array}$ & $\begin{array}{c}\text { 根长 } \\
\text { Root length } \\
(\mathrm{cm})\end{array}$ & $\begin{array}{c}\text { 芽重 } \\
\text { Bud FW } \\
\text { (mg) }\end{array}$ & $\begin{array}{c}\text { 根重 } \\
\text { Root FW } \\
(\mathrm{mg})\end{array}$ \\
\hline $\mathrm{S} 11$ & 12 & $0.00 \pm 0.00$ & $78.67 \pm 2.31$ & $0.35 \pm 0.08$ & $2.05 \pm 0.18$ & $1.33 \pm 0.34$ & $11.67 \pm 1.63$ \\
\hline \multirow[t]{4}{*}{$\mathrm{S} 12$} & 25 & $84.00 \pm 2.43$ & $89.33 \pm 2.31$ & $10.13 \pm 0.90$ & $14.78 \pm 2.05$ & $111.00 \pm 18.22$ & $50.00 \pm 7.95$ \\
\hline & 20 & $61.33 \pm 5.13$ & $86.67 \pm 2.31$ & $6.98 \pm 1.01$ & $9.73 \pm 1.41$ & $82.33 \pm 8.24$ & $56.00 \pm 10.08$ \\
\hline & 16 & $27.33 \pm 2.31$ & $84.00 \pm 4.00$ & $2.10 \pm 0.26$ & $5.67 \pm 0.81$ & $27.33 \pm 4.13$ & $36.00 \pm 5.73$ \\
\hline & 12 & $0.00 \pm 0.00$ & $81.33 \pm 2.31$ & $0.68 \pm 0.10$ & $2.10 \pm 0.22$ & $6.17 \pm 1.17$ & $13.33 \pm 2.07$ \\
\hline \multirow[t]{4}{*}{$\mathrm{S} 13$} & 25 & $87.33 \pm 5.03$ & $89.33 \pm 3.06$ & $8.97 \pm 1.34$ & $13.22 \pm 2.28$ & $78.17 \pm 13.47$ & $44.50 \pm 6.09$ \\
\hline & 20 & $78.00 \pm 5.29$ & $84.67 \pm 3.06$ & $5.87 \pm 0.48$ & $11.52 \pm 2.09$ & $59.33 \pm 9.93$ & $66.67 \pm 3.33$ \\
\hline & 16 & $5.33 \pm 0.58$ & $82.67 \pm 2.31$ & $2.03 \pm 0.24$ & $5.20 \pm 0.89$ & $24.50 \pm 4.32$ & $38.67 \pm 3.39$ \\
\hline & 12 & $0.00 \pm 0.00$ & $52.00 \pm 4.00$ & $0.10 \pm 0.00$ & $1.02 \pm 0.19$ & $0.55 \pm 0.26$ & $6.50 \pm 1.05$ \\
\hline \multirow[t]{4}{*}{$\mathrm{S} 14$} & 25 & $82.00 \pm 4.00$ & $93.33 \pm 2.31$ & $11.22 \pm 1.39$ & $14.00 \pm 1.52$ & $112.17 \pm 16.86$ & $56.67 \pm 9.27$ \\
\hline & 20 & $73.33 \pm 6.11$ & $90.67 \pm 2.31$ & $6.18 \pm 0.39$ & $10.88 \pm 1.19$ & $76.33 \pm 9.97$ & $60.17 \pm 11.32$ \\
\hline & 16 & $46.00 \pm 4.00$ & $80.00 \pm 3.46$ & $2.17 \pm 0.19$ & $6.60 \pm 0.54$ & $25.00 \pm 2.53$ & $33.17 \pm 3.06$ \\
\hline & 12 & $0.00 \pm 0.00$ & $62.00 \pm 2.00$ & $0.38 \pm 0.12$ & $1.97 \pm 0.27$ & $3.17 \pm 0.88$ & $11.33 \pm 2.16$ \\
\hline \multirow[t]{4}{*}{$\mathrm{S} 15$} & 25 & $90.67+2.31$ & $93.33 \pm 2.31$ & $9.37 \pm 0.81$ & $14.48 \pm 1.23$ & $76.67 \pm 4.59$ & $52.83 \pm 6.91$ \\
\hline & 20 & $89.33+2.31$ & $90.67 \pm 2.31$ & $5.83 \pm 0.66$ & $13.25 \pm 1.46$ & $58.33 \pm 10.13$ & $64.67 \pm 8.36$ \\
\hline & 16 & $16.00 \pm 1.00$ & $90.67 \pm 6.11$ & $2.13 \pm 0.15$ & $6.68 \pm 1.02$ & $25.33 \pm 4.72$ & $33.67 \pm 5.65$ \\
\hline & 12 & $0.00 \pm 0.00$ & $70.67 \pm 4.62$ & $0.27 \pm 0.08$ & $1.17 \pm 0.20$ & $2.17 \pm 0.75$ & $7.17 \pm 0.75$ \\
\hline \multirow[t]{4}{*}{$\mathrm{S} 16$} & 25 & $74.67 \pm 8.32$ & $89.33 \pm 2.31$ & $9.33 \pm 1.05$ & $14.80 \pm 2.21$ & $88.44 \pm 6.62$ & $51.44 \pm 9.39$ \\
\hline & 20 & $72.00 \pm 9.17$ & $81.33 \pm 6.11$ & $5.25 \pm 0.33$ & $14.20 \pm 2.62$ & $52.17 \pm 5.56$ & $67.67 \pm 6.53$ \\
\hline & 16 & $42.67 \pm 5.03$ & $80.67 \pm 7.57$ & $1.68 \pm 0.12$ & $8.93 \pm 1.28$ & $17.67 \pm 1.86$ & $47.33 \pm 7.97$ \\
\hline & 12 & $0.00 \pm 0.00$ & $56.00 \pm 4.00$ & $0.45 \pm 0.10$ & $2.10 \pm 0.25$ & $2.50 \pm 0.49$ & $12.17 \pm 0.75$ \\
\hline \multirow[t]{4}{*}{$\mathrm{S} 17$} & 25 & $78.67 \pm 6.11$ & $86.67 \pm 2.31$ & $12.42 \pm 0.69$ & $9.92 \pm 1.21$ & $101.67 \pm 12.86$ & $27.33 \pm 4.03$ \\
\hline & 20 & $73.23 \pm 5.85$ & $82.67 \pm 4.38$ & $7.22 \pm 0.63$ & $14.75 \pm 1.88$ & $72.50 \pm 11.13$ & $75.5 \pm 12.63$ \\
\hline & 16 & $58.90 \pm 3.44$ & $78.67 \pm 2.31$ & $2.14 \pm 0.34$ & $5.96 \pm 0.79$ & $21.40 \pm 4.03$ & $54.40 \pm 8.45$ \\
\hline & 12 & $0.00 \pm 0.00$ & $58.00 \pm 6.00$ & $0.50 \pm 0.13$ & $1.43 \pm 0.16$ & $3.33 \pm 1.03$ & $9.00 \pm 1.55$ \\
\hline \multirow[t]{4}{*}{$\mathrm{S} 18$} & 25 & $78.67 \pm 8.33$ & $89.33 \pm 2.31$ & $11.83 \pm 1.11$ & $13.78 \pm 2.15$ & $108.67 \pm 12.14$ & $62.50 \pm 9.46$ \\
\hline & 20 & $62.67 \pm 6.11$ & $88.00 \pm 4.00$ & $5.53 \pm 0.38$ & $9.50 \pm 1.37$ & $62.00 \pm 7.01$ & $56.50 \pm 9.73$ \\
\hline & 16 & $14.67 \pm 1.53$ & $81.33 \pm 6.11$ & $2.38 \pm 0.36$ & $6.55 \pm 0.27$ & $32.17 \pm 6.21$ & $36.33 \pm 5.20$ \\
\hline & 12 & $0.00 \pm 0.00$ & $68.00 \pm 6.93$ & $0.43 \pm 0.05$ & $1.55 \pm 0.19$ & $2.83 \pm 0.75$ & $9.50 \pm 1.87$ \\
\hline \multirow[t]{4}{*}{ S19 } & 25 & $63.33 \pm 5.03$ & $85.33 \pm 4.62$ & $8.28 \pm 0.54$ & $12.95 \pm 2.42$ & $74.67 \pm 11.52$ & $50.33 \pm 5.09$ \\
\hline & 20 & $56.00 \pm 8.00$ & $72.67 \pm 5.03$ & $7.50 \pm 0.93$ & $15.45 \pm 2.27$ & $77.17 \pm 12.91$ & $67.67 \pm 8.94$ \\
\hline & 16 & $17.33 \pm 1.53$ & $66.00 \pm 5.29$ & $2.07 \pm 0.26$ & $6.60 \pm 1.25$ & $21.00 \pm 3.03$ & $31.50 \pm 6.09$ \\
\hline & 12 & $0.00 \pm 0.00$ & $62.00 \pm 6.00$ & $0.25 \pm 0.05$ & $1.53 \pm 0.12$ & $1.83 \pm 0.68$ & $7.33 \pm 1.03$ \\
\hline \multirow[t]{4}{*}{ S20 } & 25 & $86.67 \pm 6.65$ & $88.00 \pm 8.00$ & $9.28 \pm 1.25$ & $15.87 \pm 2.83$ & $98.33 \pm 7.60$ & $32.67 \pm 1.32$ \\
\hline & 20 & $66.00 \pm 7.21$ & $80.00 \pm 4.00$ & $6.40 \pm 0.92$ & $15.38 \pm 2.63$ & $78.00 \pm 7.62$ & $82.00 \pm 10.71$ \\
\hline & 16 & $40.00 \pm 5.29$ & $77.33 \pm 3.06$ & $1.97 \pm 0.14$ & $6.95 \pm 1.09$ & $24.00 \pm 2.28$ & $35.33 \pm 4.13$ \\
\hline & 12 & $0.00 \pm 0.00$ & $74.00 \pm 2.00$ & $0.40 \pm 0.06$ & $2.37 \pm 0.35$ & $3.83 \pm 1.03$ & $15.50 \pm 1.87$ \\
\hline \multirow[t]{4}{*}{$\mathrm{S} 21$} & 25 & $86.67 \pm 6.11$ & $94.67 \pm 2.31$ & $9.27 \pm 1.04$ & $7.70 \pm 1.04$ & $87.28 \pm 9.61$ & $27.33 \pm 4.63$ \\
\hline & 20 & $80.80 \pm 3.73$ & $93.33 \pm 4.62$ & $5.98 \pm 0.67$ & $12.83 \pm 1.96$ & $58.00 \pm 11.24$ & $60.65 \pm 7.38$ \\
\hline & 16 & $72.67 \pm 5.03$ & $90.67 \pm 6.11$ & $2.50 \pm 0.28$ & $7.12 \pm 0.44$ & $26.20 \pm 3.19$ & $56.47 \pm 5.18$ \\
\hline & 12 & $0.00 \pm 0.00$ & $89.33 \pm 2.31$ & $0.30 \pm 0.06$ & $1.32 \pm 0.12$ & $2.33 \pm 0.45$ & $6.50 \pm 1.05$ \\
\hline \multirow[t]{3}{*}{ S22 } & 25 & $54.67 \pm 8.08$ & $69.33 \pm 4.62$ & $11.02 \pm 1.43$ & $14.17 \pm 2.24$ & $102.00 \pm 18.19$ & $100.67 \pm 16.85$ \\
\hline & 20 & $36.67 \pm 3.06$ & $66.67 \pm 6.11$ & $6.20 \pm 0.46$ & $10.97 \pm 1.65$ & $67.33 \pm 7.00$ & $68.83 \pm 6.52$ \\
\hline & 16 & $8.00 \pm 1.00$ & $65.33 \pm 2.31$ & $1.57 \pm 0.20$ & $4.58 \pm 0.52$ & $16.17 \pm 1.83$ & $27.67 \pm 2.07$ \\
\hline
\end{tabular}


(续表 2)

\begin{tabular}{|c|c|c|c|c|c|c|c|}
\hline $\begin{array}{c}\text { 品种 } \\
\text { Hybrid }\end{array}$ & $\begin{array}{c}\text { 温度 } \\
\text { Temperature } \\
\left({ }^{\circ} \mathrm{C}\right)\end{array}$ & $\begin{array}{c}\text { 发芽势 } \\
\text { Germination } \\
\text { potential (\%) }\end{array}$ & $\begin{array}{c}\text { 发芽率 } \\
\text { Germination } \\
\text { percent }(\%)\end{array}$ & $\begin{array}{c}\text { 芽长 } \\
\text { Bud length } \\
(\mathrm{cm})\end{array}$ & $\begin{array}{c}\text { 根长 } \\
\text { Root length } \\
(\mathrm{cm})\end{array}$ & $\begin{array}{c}\text { 芽重 } \\
\text { Bud FW } \\
(\mathrm{mg})\end{array}$ & $\begin{array}{c}\text { 根重 } \\
\text { Root FW } \\
(\mathrm{mg})\end{array}$ \\
\hline S22 & 12 & $0.00 \pm 0.00$ & $61.33 \pm 8.33$ & $0.23 \pm 0.05$ & $1.52 \pm 0.16$ & $1.17 \pm 0.27$ & $9.00 \pm 0.63$ \\
\hline \multirow[t]{4}{*}{ S23 } & 25 & $91.33 \pm 4.16$ & $96.67 \pm 1.15$ & $9.95 \pm 1.34$ & $15.23 \pm 2.92$ & $73.00 \pm 10.77$ & $46.33 \pm 6.92$ \\
\hline & 20 & $90.67 \pm 4.62$ & $96.00 \pm 4.00$ & $5.40 \pm 0.25$ & $12.43 \pm 1.26$ & $45.83 \pm 3.82$ & $58.17 \pm 4.88$ \\
\hline & 16 & $13.33 \pm 1.53$ & $94.67 \pm 2.31$ & $2.48 \pm 0.44$ & $6.85 \pm 1.07$ & $40.67 \pm 6.19$ & $33.83 \pm 6.18$ \\
\hline & 12 & $0.00 \pm 0.00$ & $52.00 \pm 8.00$ & $0.22 \pm 0.04$ & $0.95 \pm 0.15$ & $3.67 \pm 0.82$ & $5.50 \pm 0.55$ \\
\hline \multirow[t]{4}{*}{ S24 } & 25 & $76.67 \pm 5.03$ & $85.33 \pm 2.31$ & $9.83 \pm 1.14$ & $8.75 \pm 1.49$ & $79.50 \pm 12.60$ & $40.50 \pm 7.69$ \\
\hline & 20 & $66.67 \pm 6.11$ & $81.33 \pm 5.77$ & $5.68 \pm 0.62$ & $7.72 \pm 1.26$ & $54.50 \pm 7.37$ & $40.67 \pm 7.34$ \\
\hline & 16 & $16.00 \pm 1.73$ & $77.33 \pm 8.33$ & $2.05 \pm 0.21$ & $5.67 \pm 0.48$ & $25.83 \pm 2.48$ & $24.33 \pm 4.68$ \\
\hline & 12 & $0.00 \pm 0.00$ & $54.00 \pm 2.00$ & $0.23 \pm 0.05$ & $1.13 \pm 0.22$ & $1.17 \pm 0.41$ & $7.50 \pm 1.05$ \\
\hline \multirow[t]{4}{*}{$\mathrm{S} 25$} & 25 & $68.33 \pm 2.52$ & $89.33 \pm 2.31$ & $10.90 \pm 0.51$ & $16.05 \pm 2.61$ & $89.67 \pm 7.55$ & $84.67 \pm 9.14$ \\
\hline & 20 & $64.00 \pm 6.00$ & $89.33 \pm 6.11$ & $6.22 \pm 0.58$ & $14.17 \pm 1.87$ & $64.50 \pm 6.44$ & $98.50 \pm 18.25$ \\
\hline & 16 & $60.00 \pm 3.46$ & $86.67 \pm 6.11$ & $2.68 \pm 0.19$ & $7.68 \pm 0.61$ & $33.00 \pm 2.53$ & $46.17 \pm 5.81$ \\
\hline & 12 & $0.00 \pm 0.00$ & $57.33 \pm 4.62$ & $0.15 \pm 0.05$ & $1.25 \pm 0.08$ & $1.58 \pm 0.49$ & $8.83 \pm 1.17$ \\
\hline \multirow[t]{4}{*}{ S26 } & 25 & $89.33 \pm 8.33$ & $92.00 \pm 4.00$ & $9.35 \pm 1.13$ & $16.28 \pm 3.11$ & $74.67 \pm 11.2$ & $67.33 \pm 11.93$ \\
\hline & 20 & $65.33 \pm 6.43$ & $90.67 \pm 6.11$ & $4.85 \pm 0.71$ & $10.35 \pm 1.23$ & $48.00 \pm 9.23$ & $59.00 \pm 5.59$ \\
\hline & 16 & $36.00 \pm 1.73$ & $89.33 \pm 2.31$ & $2.62 \pm 0.30$ & $6.18 \pm 0.73$ & $27.00 \pm 3.41$ & $32.33 \pm 6.02$ \\
\hline & 12 & $0.00 \pm 0.00$ & $77.33 \pm 8.33$ & $0.13 \pm 0.05$ & $0.95 \pm 0.08$ & $0.67 \pm 0.22$ & $6.83 \pm 0.75$ \\
\hline \multirow[t]{4}{*}{$\mathrm{S} 27$} & 25 & $90.67 \pm 2.31$ & $93.33 \pm 2.31$ & $8.30 \pm 1.08$ & $15.13 \pm 2.06$ & $95.17 \pm 11.34$ & $51.00 \pm 9.84$ \\
\hline & 20 & $89.33 \pm 2.31$ & $89.33 \pm 6.11$ & $7.35 \pm 1.08$ & $16.13 \pm 2.32$ & $98.50 \pm 7.92$ & $78.17 \pm 8.66$ \\
\hline & 16 & $62.00 \pm 6.00$ & $88.67 \pm 3.06$ & $2.27 \pm 0.20$ & $8.07 \pm 0.79$ & $28.67 \pm 3.93$ & $46.00 \pm 7.40$ \\
\hline & 12 & $0.00 \pm 0.00$ & $38.00 \pm 2.00$ & $0.55 \pm 0.05$ & $2.28 \pm 0.29$ & $3.50 \pm 0.84$ & $12.17 \pm 1.47$ \\
\hline \multirow[t]{4}{*}{$\mathrm{S} 28$} & 25 & $84.00 \pm 10.58$ & $88.00 \pm 6.93$ & $11.87 \pm 1.37$ & $16.00 \pm 1.11$ & $94.83 \pm 15.46$ & $61.33 \pm 4.72$ \\
\hline & 20 & $60.67 \pm 6.11$ & $86.67 \pm 6.11$ & $6.20 \pm 0.87$ & $12.07 \pm 2.01$ & $68.67 \pm 10.78$ & $67.67 \pm 11.31$ \\
\hline & 16 & $36.00 \pm 2.00$ & $85.33 \pm 6.11$ & $2.63 \pm 0.30$ & $6.83 \pm 1.04$ & $30.83 \pm 5.12$ & $35.50 \pm 6.12$ \\
\hline & 12 & $0.00 \pm 0.00$ & $78.67 \pm 2.31$ & $0.38 \pm 0.10$ & $1.93 \pm 0.34$ & $2.83 \pm 0.71$ & $11.00 \pm 1.67$ \\
\hline \multirow[t]{4}{*}{ S29 } & 25 & $82.67 \pm 4.16$ & $88.00 \pm 4.00$ & $10.73 \pm 1.54$ & $8.62 \pm 1.40$ & $80.83 \pm 10.32$ & $32.67 \pm 4.80$ \\
\hline & 20 & $80.57 \pm 3.44$ & $84.00 \pm 6.93$ & $5.15 \pm 0.34$ & $10.85 \pm 1.03$ & $39.33 \pm 4.89$ & $47.83 \pm 7.47$ \\
\hline & 16 & $73.33 \pm 2.31$ & $82.67 \pm 4.62$ & $1.76 \pm 0.14$ & $6.44 \pm 0.83$ & $15.20 \pm 2.48$ & $35.20 \pm 3.19$ \\
\hline & 12 & $0.00 \pm 0.00$ & $66.67 \pm 6.11$ & $0.25 \pm 0.05$ & $1.65 \pm 0.27$ & $1.33 \pm 0.41$ & $8.33 \pm 1.03$ \\
\hline \multirow[t]{4}{*}{$\mathrm{S} 30$} & 25 & $83.33 \pm 7.02$ & $86.67 \pm 6.11$ & $9.40 \pm 0.73$ & $9.62 \pm 1.06$ & $93.00 \pm 8.10$ & $20.67 \pm 3.78$ \\
\hline & 20 & $78.30 \pm 5.00$ & $85.33 \pm 8.33$ & $6.25 \pm 0.86$ & $16.78 \pm 2.67$ & $69.17 \pm 8.06$ & $86.17 \pm 16.46$ \\
\hline & 16 & $70.00 \pm 8.00$ & $84.00 \pm 4.00$ & $2.16 \pm 0.24$ & $7.94 \pm 1.04$ & $23.00 \pm 3.90$ & $45.20 \pm 7.08$ \\
\hline & 12 & $0.00 \pm 0.00$ & $56.00 \pm 4.00$ & $0.57 \pm 0.08$ & $1.93 \pm 0.37$ & $5.67 \pm 1.21$ & $14.33 \pm 1.63$ \\
\hline 品种 & $F$-value & 42.97 & 42.84 & 7.28 & 16.59 & 16.3 & 24.31 \\
\hline Hybrid(H) & $P$-value & $* *$ & $* *$ & $* *$ & $* *$ & $* *$ & $* *$ \\
\hline 处理 & $F$ value & 3919.17 & 397.94 & 7772.28 & 2495.72 & 4756.06 & 2039.14 \\
\hline Treatment(T) & $P$-value & $* *$ & $* *$ & $* *$ & $* *$ & $* *$ & $* *$ \\
\hline \multirow[t]{2}{*}{$\mathrm{H} \times \mathrm{T}$} & $F$ value & 17.42 & 7.62 & 8.09 & 10.44 & 7.08 & 21.35 \\
\hline & $P$-value & $* *$ & $* *$ & $* *$ & $* *$ & $* *$ & $* *$ \\
\hline \multicolumn{2}{|c|}{ 变异系数 CV (\%) } & 74.29 & 17.34 & 82.17 & 60.62 & 78.02 & 59.88 \\
\hline
\end{tabular}

*显著水平 $(P<0.05) ; * *$ 极显著水平 $(P<0.01)$ 。

$\mathrm{CV}$ : coefficient of variation; *: significantly different between treatments at $P<0.05$; **: significantly different between treatments at $P<$ 0.01 . 
平，说明高粱的发芽特性与品种有关; 温度对所有 指标的影响, 差异均达到极显著水平, 说明温度是 影响发芽指标的关键因素; 温度和品种的互作对发 芽指标的影响, 差异也达到极显著水平, 说明萌发 期不同品种对温度变化的响应存在差异。高粱的发 芽势是受温度影响较大的一个指标, 变异系数为 $74.29 \%$, 发芽势以 $25^{\circ} \mathrm{C}$ 时辽杂 27 最大 $(97.33 \%)$, 本 试验中所有品种的发芽势在 $12^{\circ} \mathrm{C}$ 下均为零; 不同品 种的发芽率受温度的影响相对较小, 变异系数为 $17.34 \%$, 以 $25^{\circ} \mathrm{C}$ 时辽杂 27 最高 $(98.67 \%)$, 以 $12^{\circ} \mathrm{C}$ 时 的辽粘 3 号最低 $(10.67 \%)$; 不同品种的芽长受温度 影响较大, 变异系数达到 $82.17 \%$, 以 $25^{\circ} \mathrm{C}$ 时的吉杂 152 最大 $(12.42 \mathrm{~cm})$, 以 $12^{\circ} \mathrm{C}$ 的辽粘 3 号和吉杂 127 最小 $(0.10 \mathrm{~cm})$; 温度对不同品种根长影响也较大, 变异系数为 $60.62 \%$, 以 $20^{\circ} \mathrm{C}$ 的赤杂 101 最大 $(16.78 \mathrm{~cm}), 12^{\circ} \mathrm{C}$ 辽粘 3 号最小 $(0.72 \mathrm{~cm})$; 芽重和根
重的变化与芽长和根长的变化相似, 变异系数分别 为 $78.02 \%$ 和 $59.88 \%$, 芽重和根重以 $25^{\circ} \mathrm{C}$ 的吉杂 136 和 $407 / \mathrm{M} 5$ 最大, 分别为 $112.17 \mathrm{mg}$ 和 $100.67 \mathrm{mg}$; $12^{\circ} \mathrm{C}$ 的辽粘 3 号最小, 分别为 $0.48 \mathrm{mg}$ 和 $3.33 \mathrm{mg}$ 。

为了比较不同温度对高粱萌发指标的影响, 将 4 个温度下不同高粱品种的萌发指标分别进行平均 处理。从表 3 可以看出, 随萌发温度的降低, 高粱的 发芽势、发芽率、根长、芽长和芽重均呈现下降的 趋势。与 $25^{\circ} \mathrm{C}$ 时相比, $20^{\circ} \mathrm{C} 、 16^{\circ} \mathrm{C}$ 和 $12^{\circ} \mathrm{C}$ 时高粱的 发芽率分别下降 3.29\%、6.43\%和 $25.39 \%$; 芽长分别 下降 $41.00 \%$ 、 $78.07 \%$ 和 $96.56 \%$; 随着萌发温度的降 低, 高粱的根重表现为先增加后降低的趋势, 在 $20^{\circ} \mathrm{C}$ 时高粱的根重达到最大, 然后随温度的降低呈 下降的趋势。高粱的根芽长度比和根芽重量比均随 萌发温度的下降呈增加的趋势, 说明高粱芽与根相 比对低温更加敏感。

表 3 温度对发芽指标的影响

Table 3 Effects of temperature on germination indicators

\begin{tabular}{llccc}
\hline \multicolumn{1}{c}{ 性状 Trait } & \multicolumn{1}{c}{$25^{\circ} \mathrm{C}$} & $20^{\circ} \mathrm{C}$ & $16^{\circ} \mathrm{C}$ & $12^{\circ} \mathrm{C}$ \\
\hline 发芽势 Germination potential (\%) & $79.64 \pm 11.73 \mathrm{a}$ & $69.73 \pm 15.26 \mathrm{~b}$ & $37.32 \pm 24.57 \mathrm{c}$ & $0.00 \pm 0.00 \mathrm{~d}$ \\
发芽率 Germination percent (\%) & $88.55 \pm 6.92 \mathrm{a}$ & $85.63 \pm 8.38 \mathrm{~b}$ & $82.85 \pm 9.34 \mathrm{c}$ & $66.07 \pm 18.19 \mathrm{~d}$ \\
芽长 Bud length (cm) & $10.17 \pm 1.15 \mathrm{a}$ & $6.00 \pm 0.90 \mathrm{~b}$ & $2.23 \pm 0.36 \mathrm{c}$ & $0.35 \pm 0.16 \mathrm{~d}$ \\
根长 Root length (cm) & $12.54 \pm 3.01 \mathrm{a}$ & $12.38 \pm 2.39 \mathrm{a}$ & $6.48 \pm 1.01 \mathrm{~b}$ & $1.55 \pm 0.46 \mathrm{c}$ \\
芽重 Bud FW (mg) & $90.25 \pm 12.66 \mathrm{a}$ & $62.58 \pm 12.88 \mathrm{~b}$ & $24.95 \pm 5.56 \mathrm{c}$ & $2.56 \pm 1.70 \mathrm{~d}$ \\
根重 Root FW (mg) & $48.28 \pm 20.66 \mathrm{~b}$ & $63.76 \pm 12.48 \mathrm{a}$ & $37.16 \pm 9.57 \mathrm{c}$ & $9.41 \pm 2.96 \mathrm{~d}$ \\
根芽比(长度) Root/bud (length) & $1.01 \pm 0.32 \mathrm{~d}$ & $1.91 \pm 0.33 \mathrm{c}$ & $2.62 \pm 0.83 \mathrm{~b}$ & $5.01 \pm 1.79 \mathrm{a}$ \\
根芽比(鲜重) Root/bud (FW) & $0.54 \pm 0.23 \mathrm{c}$ & $1.04 \pm 0.21 \mathrm{bc}$ & $1.57 \pm 0.56 \mathrm{~b}$ & $4.80 \pm 2.41 \mathrm{a}$ \\
\hline
\end{tabular}

同一行中标以相同字母的值在 0.05 水平差异不显著。

Values followed by the same letters at the same row are not significantly different at $P<0.05$.

为了比较不同品种对温度变化的响应差异, 将 $20^{\circ} \mathrm{C} 、 16^{\circ} \mathrm{C} 、 12^{\circ} \mathrm{C}$ 的萌发指标分别以 $25^{\circ} \mathrm{C}$ 为对照作 比并加权平均, 得到不同指标的相对值如表 4 所示。 从不同品种相对值的均值看, 发芽势、芽长、芽重
的相对值较小, 说明这 3 个指标受温度的影响较大； 从萌发指标的相对值在不同品种之间的变异来看, 发芽势、根长、根重的变异系数较大, 说明这 3 个 指标对温度的响应在不同品种之间差异较大。

表 4 不同高粱品种萌发特性相对值

Table 4 Relative value of all traits in different sorghum hybrids (\%)

\begin{tabular}{ccccccc}
\hline $\begin{array}{c}\text { 品种 } \\
\text { Hybrid }\end{array}$ & $X_{1}$ & $X_{2}$ & $X_{3}$ & $X_{4}$ & $X_{5}$ & $X_{6}$ \\
\hline S1 & $31.21 \pm 4.75 \mathrm{gh}$ & $92.95 \pm 5.53 \mathrm{ac}$ & $22.01 \pm 1.03 \mathrm{ce}$ & $39.40 \pm 3.01 \mathrm{fi}$ & $20.91 \pm 2.07 \mathrm{kn}$ & $37.66 \pm 1.90 \mathrm{no}$ \\
S2 & $44.30 \pm 1.43 \mathrm{ad}$ & $90.86 \pm 1.26 \mathrm{ad}$ & $19.26 \pm 1.07 \mathrm{fj}$ & $67.62 \pm 3.94 \mathrm{a}$ & $21.97 \pm 1.09 \mathrm{ik}$ & $155.08 \pm 9.07 \mathrm{~b}$ \\
S3 & $28.01 \pm 6.37 \mathrm{hk}$ & $96.05 \pm 2.89 \mathrm{a}$ & $21.04 \pm 1.02 \mathrm{cf}$ & $39.17 \pm 4.17 \mathrm{gi}$ & $25.39 \pm 0.82 \mathrm{dg}$ & $40.46 \pm 3.09 \mathrm{mn}$ \\
S4 & $38.89 \pm 0.93 \mathrm{df}$ & $70.77 \pm 1.03 \mathrm{ij}$ & $18.76 \pm 1.07 \mathrm{gk}$ & $61.19 \pm 2.16 \mathrm{bc}$ & $25.75 \pm 0.58 \mathrm{df}$ & $166.71 \pm 16.62 \mathrm{~b}$ \\
S5 & $8.02 \pm 1.12 \mathrm{o}$ & $51.71 \pm 4.40 \mathrm{k}$ & $13.56 \pm 0.22 \mathrm{~m}$ & $23.08 \pm 0.79 \mathrm{~m}$ & $16.41 \pm 0.45 \mathrm{o}$ & $27.46 \pm 1.81 \mathrm{o}$ \\
S6 & $24.75 \pm 2.18 \mathrm{il}$ & $92.52 \pm 2.05 \mathrm{ac}$ & $17.31 \pm 0.55 \mathrm{jl}$ & $30.19 \pm 1.25 \mathrm{kl}$ & $22.10 \pm 0.64 \mathrm{hk}$ & $40.37 \pm 3.71 \mathrm{mn}$ \\
S7 & $48.49 \pm 1.16 \mathrm{a}$ & $95.80 \pm 2.53 \mathrm{a}$ & $19.53 \pm 0.69 \mathrm{fi}$ & $56.33 \pm 4.38 \mathrm{~cd}$ & $21.05 \pm 0.58 \mathrm{kn}$ & $128.91 \pm 4.45 \mathrm{c}$ \\
S8 & $45.63 \pm 1.70 \mathrm{ac}$ & $93.50 \pm 0.82 \mathrm{ac}$ & $17.14 \pm 0.90 \mathrm{kl}$ & $66.63 \pm 4.64 \mathrm{ab}$ & $18.68 \pm 2.28 \mathrm{mo}$ & $115.11 \pm 9.18 \mathrm{de}$ \\
\hline
\end{tabular}


(续表 4)

\begin{tabular}{|c|c|c|c|c|c|c|}
\hline $\begin{array}{c}\text { 品种 } \\
\text { Hybrid } \\
\end{array}$ & $X_{1}$ & $X_{2}$ & $X_{3}$ & $X_{4}$ & $X_{5}$ & $X_{6}$ \\
\hline S9 & $22.42 \pm 1.76 \mathrm{kn}$ & $97.80 \pm 1.26 \mathrm{a}$ & $30.00 \pm 1.66 \mathrm{a}$ & $43.07 \pm 1.58 \mathrm{eg}$ & $29.08 \pm 3.20 \mathrm{bc}$ & $84.01 \pm 2.02 \mathrm{f}$ \\
\hline $\mathrm{S} 10$ & $28.68 \pm 1.97 \mathrm{hj}$ & $81.23 \pm 5.42 \mathrm{eh}$ & $17.63 \pm 0.73$ il & $36.84 \pm 3.05 \mathrm{hj}$ & $22.66 \pm 1.11 \mathrm{gk}$ & $37.25 \pm 1.64$ no \\
\hline S11 & $23.71 \pm 4.95 \mathrm{jm}$ & $93.58 \pm 2.40 \mathrm{ac}$ & $22.67 \pm 0.42 \mathrm{c}$ & $61.45 \pm 2.31 b c$ & $23.08 \pm 1.55 \mathrm{fk}$ & $81.16 \pm 4.36 \mathrm{fg}$ \\
\hline $\mathrm{S} 12$ & $24.28 \pm 4.70$ il & $93.14 \pm 1.23 \mathrm{ac}$ & $22.84 \pm 1.23 \mathrm{c}$ & $31.90 \pm 1.65 \mathrm{jl}$ & $24.77 \pm 1.52 \mathrm{di}$ & $57.60 \pm 2.66 \mathrm{ik}$ \\
\hline S13 & $18.55 \pm 0.78 \mathrm{mn}$ & $76.40 \pm 0.97 \mathrm{hi}$ & $20.35 \pm 1.17 \mathrm{eh}$ & $32.95 \pm 1.63 \mathrm{j} 1$ & $24.85 \pm 1.39 \mathrm{dh}$ & $64.16 \pm 4.33 \mathrm{hj}$ \\
\hline S14 & $35.22 \pm 1.28 \mathrm{fg}$ & $78.54 \pm 1.01 \mathrm{fh}$ & $18.38 \pm 1.02 \mathrm{hk}$ & $36.93 \pm 1.65 \mathrm{hj}$ & $21.47 \pm 1.09 \mathrm{~km}$ & $48.95 \pm 1.77 \mathrm{kn}$ \\
\hline S15 & $24.11 \pm 1.45 \mathrm{im}$ & $86.79 \pm 2.79$ ce & $20.49 \pm 1.20 \mathrm{dg}$ & $36.09 \pm 1.70 \mathrm{hj}$ & $26.34 \pm 3.33$ ce & $50.41 \pm 1.61 \mathrm{~km}$ \\
\hline S16 & $36.88 \pm 0.51$ ef & $77.09 \pm 4.68 \mathrm{hi}$ & $18.81 \pm 0.72 \mathrm{gk}$ & $44.74 \pm 1.13$ ef & $18.90 \pm 0.70$ lo & $67.06 \pm 4.82 \mathrm{hi}$ \\
\hline S17 & $42.23 \pm 0.79$ be & $80.10 \pm 3.23 \mathrm{eh}$ & $18.39 \pm 1.21 \mathrm{gk}$ & $54.58 \pm 3.14 \mathrm{~d}$ & $21.70 \pm 1.17 \mathrm{jl}$ & $133.62 \pm 13.08 \mathrm{c}$ \\
\hline S18 & $20.87 \pm 2.42 \ln$ & $85.17 \pm 4.50 \mathrm{dg}$ & $17.14 \pm 0.80 \mathrm{kl}$ & $34.40 \pm 3.22 \mathrm{hk}$ & $21.64 \pm 1.38 \mathrm{jl}$ & $43.52 \pm 2.78 \ln$ \\
\hline S19 & $25.32 \pm 3.55$ il & $76.47 \pm 3.18 \mathrm{hi}$ & $26.48 \pm 2.07 \mathrm{~b}$ & $45.02 \pm 2.72 \mathrm{e}$ & $29.70 \pm 1.69 \mathrm{~b}$ & $52.56 \pm 3.38 \mathrm{j} 1$ \\
\hline S20 & $29.43 \pm 1.50 \mathrm{hi}$ & $86.91 \pm 4.83$ be & $22.03 \pm 1.90 \mathrm{ce}$ & $39.86 \pm 2.25 \mathrm{eh}$ & $24.66 \pm 0.93 \mathrm{di}$ & $105.15 \pm 9.62 \mathrm{e}$ \\
\hline S21 & $45.24 \pm 0.81 \mathrm{ac}$ & $95.60 \pm 2.08 \mathrm{a}$ & $22.51 \pm 0.97 \mathrm{~cd}$ & $70.31 \pm 5.17 \mathrm{a}$ & $23.49 \pm 1.43 \mathrm{ek}$ & $122.58 \pm 9.24 \mathrm{~cd}$ \\
\hline S22 & $17.35 \pm 1.15 \mathrm{n}$ & $91.74 \pm 3.24 \mathrm{ad}$ & $16.25 \pm 0.731$ & $30.43 \pm 1.28 \mathrm{kl}$ & $18.30 \pm 1.88$ no & $26.45 \pm 2.51 \mathrm{o}$ \\
\hline $\mathrm{S} 23$ & $23.19 \pm 2.03 \mathrm{jm}$ & $76.91 \pm 4.72 \mathrm{hi}$ & $19.48 \pm 0.88 \mathrm{fi}$ & $33.61 \pm 3.57 \mathrm{jk}$ & $32.70 \pm 1.20 \mathrm{a}$ & $53.46 \pm 1.37 \mathrm{jl}$ \\
\hline S24 & $22.95 \pm 2.69 \mathrm{kn}$ & $78.29 \pm 3.76 \mathrm{gh}$ & $18.84 \pm 1.09 \mathrm{gk}$ & $44.66 \pm 4.60 \mathrm{eg}$ & $24.42 \pm 1.94 \mathrm{dj}$ & $47.66 \pm 2.84 \mathrm{kn}$ \\
\hline S25 & $47.03 \pm 2.22 \mathrm{ab}$ & $81.72 \pm 4.59 \mathrm{eh}$ & $19.41 \pm 1.12 \mathrm{fj}$ & $36.47 \pm 3.29 \mathrm{hj}$ & $26.44 \pm 1.21 \mathrm{~cd}$ & $44.58 \pm 2.38 \ln$ \\
\hline S26 & $26.94 \pm 1.76 \mathrm{hk}$ & $91.03 \pm 2.44 \mathrm{ad}$ & $19.60 \pm 0.82 \mathrm{fi}$ & $27.61 \pm 2.67 \mathrm{~lm}$ & $24.39 \pm 1.28 \mathrm{dj}$ & $37.45 \pm 2.57$ no \\
\hline S27 & $41.02 \pm 1.86 \mathrm{ce}$ & $68.97 \pm 1.86 \mathrm{j}$ & $28.75 \pm 1.22 \mathrm{a}$ & $44.89 \pm 1.61$ ef & $31.04 \pm 1.15 \mathrm{ab}$ & $70.68 \pm 5.12 \mathrm{gh}$ \\
\hline S28 & $27.6 \pm 1.99 \mathrm{hk}$ & $93.78 \pm 4.45 \mathrm{ab}$ & $18.61 \pm 1.25 \mathrm{gk}$ & $33.86 \pm 3.30 \mathrm{ik}$ & $25.69 \pm 1.49 \mathrm{df}$ & $48.11 \pm 4.41 \mathrm{kn}$ \\
\hline S29 & $47.65 \pm 1.22 \mathrm{ab}$ & $85.40 \pm 2.34 \mathrm{df}$ & $15.62 \pm 1.33 \mathrm{~lm}$ & $57.83 \pm 3.06 \mathrm{~cd}$ & $16.06 \pm 0.85 \mathrm{o}$ & $75.70 \pm 3.67 \mathrm{fh}$ \\
\hline $\mathrm{S} 30$ & $45.37 \pm 0.57 \mathrm{ac}$ & $81.67 \pm 0.63 \mathrm{eh}$ & $22.81 \pm 1.40 \mathrm{c}$ & $69.31 \pm 3.67 \mathrm{a}$ & $24.85 \pm 1.47 \mathrm{dh}$ & $184.80 \pm 13.69 \mathrm{a}$ \\
\hline 平均值 Mean & 31.51 & 84.75 & 20.19 & 44.35 & 23.62 & 74.96 \\
\hline $\begin{array}{c}\text { 变异范围 } \\
\text { Range }\end{array}$ & $8.02-48.49$ & $51.71-97.80$ & $13.56-30.00$ & $23.08-70.31$ & $16.06-32.70$ & $26.45-184.80$ \\
\hline $\begin{array}{c}\text { 变异系数 } \\
\text { CV (\%) }\end{array}$ & 34.40 & 12.07 & 17.77 & 30.75 & 16.77 & 58.36 \\
\hline
\end{tabular}

$X_{1} 、 X_{2} 、 X_{3} 、 X_{4} 、 X_{5}$ 和 $X_{6}$ 分别代表相对发芽势、相对发芽率、相对芽长、相对根长、相对芽重和相对根重。同一列中标以相同字母 的值 0.05 水平差异不显著。

$X_{1}, X_{2}, X_{3}, X_{4}, X_{5}$, and $X_{6}$ represent relative germination potential, relative germination rate, relative bud length, relative root length, relative bud fresh weight and relative root fresh weight, respectively. Values followed by the same letters within the same column are not significantly different at $P<0.05$.

\section{2 萌发指标相对值之间的相关性分析}

相关分析表明, 相对发芽势与相对根长呈极显 著正相关 $(r=0.74, P<0.01)$, 相对发芽势与相对根重 呈极显著正相关 $(r=0.66, P<0.01)$, 表明根系生长对 高粱种子发芽势具有一定的正向效应。相对芽长与 相对芽重呈极显著正相关 $(r=0.71, P<0.01)$, 相对根 长与相对根重之间呈极显著正相关 $(r=0.85, P<0.01)$, 说明在不同温度下高粱种子萌发形态与其重量指标 之间具有一致性。相对发芽率与萌发指标相对值之 间的相关性均未达到显著水平(表 5)。

\section{3 高粱萌发期耐低温特性的主成分分析}

主成分分析可以基于原先提出的所有变量情况下, 进行精简, 删除多余重复的变量, 建立尽可能少的新变 量, 这些新的变量两两不相关, 可以更加全面准确地反 映高粱萌发期的耐低温能力。对本研究中 30 个高粱品 种在不同温度下萌发指标的相对值进行主成分分析。前 3 个主成分的贡献率分别为 $43.23 \% 、 28.56 \%$ 和 $16.21 \%$, 累计贡献率达到 $87.99 \%$ (附表 1), 考虑到累计贡献率大 于 $80 \%$ 的原则, 说明前 3 个主成分已经足以代表该组数 据的基本信息, 符合主成分分析的要求。 
表 5 萌发期低温胁迫下各性状的相关系数

Table 5 Correlation coefficients among all germination traits of sorghum under low temperature

\begin{tabular}{cccccc}
\hline $\begin{array}{c}\text { 萌发指标 } \\
\text { Germination traits }\end{array}$ & $X_{1}$ & $X_{2}$ & $X_{3}$ & $X_{4}$ & $X_{5}$ \\
\hline$X_{1}$ & 1 & & & & \\
$X_{2}$ & 0.20 & 1 & & & \\
$X_{3}$ & 0.06 & 0.20 & 1 & 1 & 1 \\
$X_{4}$ & $0.74^{* *}$ & 0.21 & 0.17 & -0.10 & 0.01 \\
$X_{5}$ & -0.09 & -0.02 & $0.71^{* *}$ & $0.85^{* *}$ & 1 \\
$X_{6}$ & $0.66^{* *}$ & 0.08 & 0.17 & & \\
\hline
\end{tabular}

$X_{1} 、 X_{2} 、 X_{3} 、 X_{4} 、 X_{5}$ 和 $X_{6}$ 分别代表相对发芽势、相对发芽率、相对芽长、相对根长、相对芽重和相对根重。 ${ }^{*}$ 和 ${ }^{* *}$ 分别表示 0.05 和 0.01 的显著水平。

$X_{1}, X_{2}, X_{3}, X_{4}, X_{5}$, and $X_{6}$ represent relative germination potential, relative germination rate, relative bud length, relative root length, relative bud fresh weight and relative root fresh weight, respectively. ${ }^{*}$ and ${ }^{* *}$ indicate significance at $P<0.05$ and $P<0.01$, respectively.

从各个萌发指标的载荷矩阵分析, 相对根长、 相对根重、相对发芽势在第 I 主成分中的载荷比较 大, 说明第一主成分主要反映的是根部性状的重要 指标。在第 II 主成分中相对芽长和相对芽重的载荷 较大, 说明第二主成分主要反映的是芽生长状况的 重要指标。第 III 主成分中相对发芽率的载荷较 大, 说明第三主成分主要反映的是种子发芽的状 况(附表 2)。

根据各因子的得分系数矩阵(附表 3), 将原先的 6 个指标转化为 3 个主成分, 3 个主成分的得分公式 如下。

$$
\begin{array}{cr}
Y_{1}=0.329 X_{1}+0.118 X_{2}+0.105 X_{3}+0.363 X_{4}+ \\
0.004 X_{5}+0.346 X_{6} \\
Y_{2}=-0.097 X_{1}+0.079 X_{2}+0.523 X_{3}-0.065 X_{4}+ \\
0.538 X_{5}-0.031 X_{6} \\
Y_{3}=-0.026 X_{1}+0.961 X_{2}+0.031 X_{3}-0.064 X_{4}- \\
0.198 X_{5}-0.244 X_{6}
\end{array}
$$

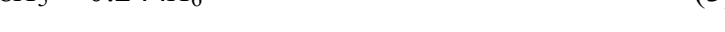

根据各主成分的贡献值在累计贡献率中所占的比值 进行加权, 各品种的综合得分公式如下。

$$
Y=0.491 Y_{1}+0.325 Y_{2}+0.184 Y_{3}
$$

计算后 $Y$ 值见附表 4, $Y$ 值越大, 表明品种的耐 低温萌发能力越强。因此, 不同高粱品种的耐低温 特性为 $\mathrm{S} 30>\mathrm{S} 2>\mathrm{S} 21>\mathrm{S} 7>\mathrm{S} 8>\mathrm{S} 4>\mathrm{S} 17>\mathrm{S} 9>$ $\mathrm{S} 11>\mathrm{S} 20>\mathrm{S} 29>\mathrm{S} 27>\mathrm{S} 12>\mathrm{S} 3>\mathrm{S} 28>\mathrm{S} 1>\mathrm{S} 19$ $>\mathrm{S} 16>\mathrm{S} 25>\mathrm{S} 15>\mathrm{S} 26>\mathrm{S} 6>\mathrm{S} 14>\mathrm{S} 23>\mathrm{S} 24>$ $\mathrm{S} 13>\mathrm{S} 18>\mathrm{S} 10>\mathrm{S} 22>\mathrm{S} 5$ 。

\section{4 基于主成分综合得分的聚类分析}

根据主成分分析得出的 Y 值进行聚类分析, 利 用系统分类的组内联接法将 30 个高粱品种分为四 大类(表 6), 第一类为低温高度敏感品种, 仅含辽粘 3 号(S5) 1 个品种; 第二类为低温敏感品种, 包含济 粱 1 号(S1)和冀酿 1 号(S3)等 18 个品种; 第三类为 耐低温品种, 包含辽杂 $36(\mathrm{~S} 9)$ 和吉杂 $152(\mathrm{~S} 17)$ 等 5 个品种; 第四类为极耐低温品种, 包含赤杂 101(S30) 和济粱 2 号(S2)等 6 个品种。

表 6 不同高粱品种耐低温特性聚类结果

Table 6 Classification of cold-resistance of sorghum hybrids based on clustering analysis

\begin{tabular}{ll}
\hline $\begin{array}{c}\text { 温度敏感类别 } \\
\text { Type of temperature-sensitivity }\end{array}$ & \multicolumn{1}{c}{$\begin{array}{c}\text { 品种 } \\
\text { Hybrid }\end{array}$} \\
\hline 高度低温敏感 Highly cold-sensitive & $\mathrm{S} 5$ \\
低温敏感 Cold-sensitive & $\mathrm{S} 1, \mathrm{~S} 3, \mathrm{~S} 6, \mathrm{~S} 10, \mathrm{~S} 12, \mathrm{~S} 13, \mathrm{~S} 14, \mathrm{~S} 15, \mathrm{~S} 16, \mathrm{~S} 18, \mathrm{~S} 19, \mathrm{~S} 22, \mathrm{~S} 23, \mathrm{~S} 24, \mathrm{~S} 25, \mathrm{~S} 26, \mathrm{~S} 27, \mathrm{~S} 28$ \\
低温不敏感 Cold-tolerance & $\mathrm{S} 9, \mathrm{~S} 11, \mathrm{~S} 17, \mathrm{~S} 19, \mathrm{~S} 20$ \\
低温极不敏感 Highly cold-tolerance & $\mathrm{S} 2, \mathrm{~S} 4, \mathrm{~S} 7, \mathrm{~S} 8, \mathrm{~S} 21, \mathrm{~S} 30$ \\
\hline
\end{tabular}

\section{3 讨论}

早春的低温通常会造成高粱种子萌发困难, 田 间出苗不齐, 进而影响高粱群体的建成。中国高粱 具有较强的耐低温特性 ${ }^{[20]}$, 但目前栽培的高粱品种 主要为杂交种, 杂交种的母本都是国外类型或者倾
国外类型, 耐低温能力相对较差 ${ }^{[21]}$ 。本研究的试验 材料均来源于中国不同生态区，评价与鉴定其萌发 期耐低温特性, 对高粱生产栽培具有一定的理论和 实践意义。从试验结果看, 各生态区均有低温极不 敏感品种及低温敏感品种, 说明高粱杂交种的耐低 
温特性与地区分布的相关性不大。一方面可能是由 于高粱的生态适应性较强，各地区间育种材料的交 流较为频繁, 使各生态区均有不同耐低温类型的高 粱种质; 另一方面, 现阶段高粱品种的选择多注重 产量和适宜机械化的株型性状, 生产和育种中多通 过调节播期来避免早春低温对高粱出苗的影响, 而 对耐低温性状的关注不够。

本研究发现, 种子萌发过程中不同萌发指标在 不同程度上均受到低温的影响, 与发芽率相比, 高 粱芽的生长受到低温的影响更加明显(表 3), 说明高 粱芽的生长比发芽对低温更加敏感。该结果与 Balota 等 ${ }^{[22]}$ 研究相一致, 说明高粱种子萌发过程中 的不同阶段对温度的敏感性不同。常博文等 ${ }^{[23]}$ 研究 也发现, 低温对不同花生品种的发芽率影响不显著, 但显著影响花生芽的伸长。因此, 在鉴定高粱耐低 温萌发能力时，只关注低温对高粱发芽率的影响具 有一定的局限性。本研究还发现高粱种子在萌发过 程中, 根与芽对低温的耐受性也存在一定差异, 虽 然低温显著抑制高粱芽和根的生长, 但高粱的根芽 长度比和根芽重量比随着萌发温度的降低均呈增加 的趋势, 表明芽的生长比根的生长对萌发期低温更 加敏感。张海艳 ${ }^{[24]}$ 在玉米上也发现萌发期的低温首 先抑制芽的生长其次才会抑制根的生长。这极有可 能与种子内部的激素含量变化有关 ${ }^{[25]}$, 但如何变化 还有待进一步研究。

高粱的萌发特性是基因和环境互作的多种萌发 指标的综合表现 ${ }^{[26-27]}$ 。选取有代表性的指标，建立 客观的评价体系, 对高粱品种萌发期耐低温特性的 评价和耐低温品种的鉴定都非常重要。前人多以种 子的萌发率或出苗率作为评价作物发芽特性的指 标 ${ }^{[28-30]}$ 。单一的指标评价作物的萌发特性具有一定 的局限性, 应选取多项指标综合评定。前人对不同 试验水平处理多将抗逆相对值平均处理 ${ }^{[31-32]}$, 但不 同水平的低温对高粱萌发指标的效应是不相同的, 因此, 本研究根据不同低温程度, 将不同指标相对 值赋予不同的权重系数进行加权平均, 这样得到的 相对值更能体现高粱品种对不同温度水平的响应差 异。同时, 本研究选取了相对发芽势、相对发芽率、 相对芽长、相对根长、相对芽重、相对根重 6 个指 标对高粱的萌发期耐低温特性综合评价，尽管这些 观测指标之间存在一定的相关性, 但是我们采用上 述加权平均的方式耦合主成分分析, 通过对多项指 标降维处理, 篮选到 3 个主成分, 其中相对芽长、相
对根长和相对发芽率载荷最大, 可代表处理间变异 的大部分信息, 因此, 这 3 个指标可作为高粱萌发 期耐低温特性评价的主要鉴定指标。基于主成分分 析对每个高粱品种的耐低温萌发特性综合评分, 通 过聚类分析将综合评分分类, 从排序和分类结果上 看, 高粱萌发的耐低温差异与试验过程中性状表现 趋势(表 2)基本一致, 说明本研究采用的加权方式耦 合基于主成分与聚类分析对高粱萌发期耐低温性评 价具有较好的可靠性。

前人研究发现, 针对低温对高粱萌发能力的影 响, 在培养箱进行的试验与大田试验具有一致的效 果, 在 2 种环境中发芽指标之间具有显著的相关性, 所以培养箱内的发芽试验可以模拟大田的低温萌发 试验 ${ }^{[33]}$ 。大田试验通过不同播期, 根据气温的差异 研究高粱耐低温萌发能力 ${ }^{[34]}$, 由于不可控因素较多 (例如, 温度易受环境及年际间的影响), 试验结果易 产生较大误差。在气候箱内通过培养血进行发芽试 验, 可对萌发期的温度精确控制, 消除高粱种子在 大田萌发过程中不可控因素的影响, 更加精确地研 究萌发期温度对高粱萌发能力的影响。本研究篮选 出的耐低温品种及鉴定指标今后可以在大田试验中 进一步验证。我们对篮选出的极端品种在不同温度 下进行了多次重复的发芽试验, 结果具有很好的重 现性。

\section{4 结论}

高粱萌发过程中低温显著抑制了高粱种子芽和 根的伸长, 且芽对低温的敏感性高于根; 相对芽长、 相对根长和相对发芽率可作为高粱萌发期耐低温的 䇻选指标; 根据高粱品种萌发期耐低温的能力, 可 分为四大类, 辽粘 3 号萌发期极不耐低温, 济粱 1 号 等 18 个品种对低温敏感, 辽杂 36 等 5 个品种对低 温不敏感, 赤杂 101 等 6 个品种对萌发期低温具有 较强的耐性。

\section{References}

[1] Huang R D. Research progress on plant tolerance to soil salinity and alkalinity in sorghum. $J$ Integr Agric, 2018, 17: 739-746.

[2] Tiryaki I, Andrews D J. Germination and seedling cold tolerance in sorghum: II. Parental lines and hybrids. Agron J, 2001, 93: 1391-1397.

[3] Peacock J M. Response and tolerance of sorghum to temperature stress. In: House L R, Mughogho L K, Peacock J M, eds. Sorghum in the Eighties Proceedings of the International Symposium on Sorghum. Patancheru, India: International Crops Research Institute for the Semi-Arid Tropics. 1982. pp 143-159. 
[4] Yu J, Tuinstra M R. Genetic analysis of seedling growth under cold temperature stress in grain sorghum. Crop Sci, 2001, 41: 1438-1443.

[5] Bekele W A, Fiedler K, Shiringani A, Schnaubelt D, Windpassinger S, Uptmoor R, Friedt W, Snowdon R J. Unravelling the genetic complexity of sorghum seedling development under low-temperature conditions. Plant Cell Environ, 2014, 37: 707-723.

[6] Ma Z, Bykova N V, Igamberdiev A U. Cell signaling mechanisms and metabolic regulation of germination and dormancy in barley seeds. Crop J, 2017, 5: 459-477.

[7] Aoki N, Scofield G N, Wang X D, Offler H E, Patrick J W, Furbank R T. Pathway of sugar transport in germinating wheat seeds. Plant Physiol, 2006, 141: 1255-1263.

[8] Hong Y F, Ho T H D, Wu C F, Ho S L, Yeh R H, Lu C A, Chen P W, Yu L C, Chao A, Yu A M. Convergent starvation signals and hormone crosstalk in regulating nutrient mobilization upon germination in cereals. Plant Cell, 2012, 24: 2857-2873.

[9] Ma Z, Marsolais F, Bernards M A, Sumarahb M W, Bykovad N V, Lgamberdiev A U. Glyoxylate cycle and metabolism of organic acids in the scutellum of barley seeds during germination. Plant Sci, 2016, 248: 37-44.

[10] 鲜孟筑, 杨萍, 胡立勇, 徐正华. 油菜种子萌发成苗期耐低温 性评价. 作物杂志, 2015, (5): 116-122.

Xian M Z, Yang P, Hu L Y, Xu Z H. Comprehensive evaluation of low temperature tolerance in rapeseed during germination and emergence periods. Crops, 2015, (5): 116-122 (in Chinese with English abstract).

[11] Thompson K, Grime J P, Mason G. Seed germination in response to diurnal fluctuations of temperature. Nature, 1977, 267: 147-149.

[12] Upadhyaya H D, Wang Y H, Sastry D V S S R, Dwivedi S L, Prasad P V V, Burrell A M, Klein R R, Morris G P, Klein P E. Association mapping of germinability and seedling vigor in sorghum under controlled low-temperature conditions. Genome, 2016, 59: 137-145.

[13] Franks C D, Burow G B, Burke J J. A comparison of us and Chinese sorghum germplasm for early season cold tolerance. Crop Sci, 2006, 46: 1371-1376.

[14] 张丽霞, 张明政, 张灵敏, 霍岩, 王春语, 丛玲. 高粱种子萌 发期耐低温材料的篮选与鉴定. 西南农业学报, 2017, 30: $20-25$.

Zhang L X, Zhang M Z, Zhang L M, Huo Y, Wang C Y, Cong L. Screening and identification of low-temperature resistant materials at seed stage. Southwest China J Agric Sci, 2017, 30: 20-25 (in Chinese with English abstract).

[15] 张海燕, 史红梅, 杨彬, 周忠宇, 张桂香. 高粱种子耐冷材料 篲选鉴定与利用评价. 种子, 2016, 35(3): 65-67.

Zhang H Y, Shi H M, Yang B, Zhou Z Y, Zhang G X. Utilization evaluation and screening identification on the chilling tolerance materials of sorghum seeds. Seed, 2016, 35(3): 65-67 (in Chinese with English abstract).

[16] 朱晨曦, 马艳青, 杨博智, 张竹青. 不同辣椒品种种子萌发期 耐低温性的研究. 中国蔬菜, 2015, (8): 34-38.

Zhu C X, Ma Y Q, Yang B Z, Zhang Z Q. Studies on low temperature tolerance of different pepper varieties in seed germina- tion stage. Chin Veg, 2015, (8): 34-38 (in Chinese with English abstract).

[17] 王俊娟, 王德龙, 阴祖军, 王帅, 樊伟丽, 陆许可, 穆敏, 郭丽 雪, 叶武威, 喻树迅. 陆地棉萌发至幼苗期抗冷性的鉴定. 中 国农业科学, 2016, 49: 3332-3346.

Wang J J, Wang D L, Yin Z J, Wang S, Fan W L, Lu X K, Mu M, Guo L X, Ye W W, Yu S X. Identification of the chilling resistance from germination stage to seedling stage in upland cotton. Sci Agric Sin, 2016, 49: 3332-3346 (in Chinese with English abstract).

[18] 张雪峰, 张立军, 胡滨. 玉米萌发期种子耐低温鉴定指标的篮 选. 辽宁农业科学, 2011, (1): 25-30.

Zhang X F, Zhang L J, Hu B. Effects of chilling stress on chill-resistance physiological indexes of maize germinating seeds. Liaoning Agric Sci, 2011, (1): 25-30 (in Chinese with English abstract).

[19] 高利英, 邓永胜, 韩宗福, 孔凡金, 申贵芳, 李汝忠, 尹燕枰. 黄淮棉区棉花品种种子萌发期低温耐受性评价. 棉花学报, 2018, 30: 36-44.

Gao L Y, Deng Y S, Han Z F, Kong F J, Shen G F, Li R Z, Yin Y P. Evaluation of the low-temperature tolerance of cotton varieties in the Huang-Huai region during seed germination. Cotton Sci, 2018, 30: 36-44 (in Chinese with English abstract).

[20] Lu Q S, Dahlberg J A. Chinese sorghum genetic resources. Econ Bot, 2001, 55: 401-425.

[21] 高士杰, 陈冰嬬, 李继洪, 贾俊英, 侯玉波. 中国高粱春播早 熟区雄性不育系存在的问题探讨. 吉林农业科学, 2012, 37(5): $9-11$.

Gao S J, Chen B X, Li J H, Jia J Y, Hou Y B. Discussions on problems in male sterile line of sorghum in spring seeding early-maturing region in China. J Jilin Agric Sci, 2012, 37(5): 9-11 (in Chinese with English abstract).

[22] Balota M, Payne W A, Veergoni S K, Stewrt B A, Rosenow D T. Respiration and its relationship to germination, emergence, and early growth under cool temperatures in sorghum. Crop Sci, 2010, 50: 1414-1422.

[23] 常博文, 钟鹏, 刘杰, 唐中华, 高亚冰, 于洪久, 郭炜. 低温胁 迫和赤霉素对花生种子萌发和幼苗生理响应的影响. 作物学 报, 2019, 45: 122-134.

Chang B W, Zhong P, Liu J, Tang Z H, Gao Y B, Yu H J, Guo W. Effect of low-temperature stress and gibberellin on seed germination and seedling physiological responses in peanut. Acta Agron Sin, 2019, 45: 122-134 (in Chinese with English abstract).

[24] 张海艳. 低温对鲜食玉米种子萌发及幼苗生长的影响. 植物 生理学报, 2013, 49: 347-350.

Zhang H Y. Effects of low temperature on seed germination and seedling growth of fresh corn. Acta Phytophysiol Sin, 2013, 49: 347-350 (in Chinese with English abstract).

[25] 江玲, 侯名语, 刘世家, 陈亮明, 刘喜, 翟虎渠, 万建民. 水稻 种子低温萌发生理机制的初步研究. 中国农业科学, 2005, 38: 480-485.

Jiang L, Hou M Y, Liu S J, Chen L M, Liu X, Zhai H Q, Wan J M. Physiological mechanism of seed germination (Oryza sativa L.) under low temperature. Sci Agric Sin, 2005, 38: 480-485 (in Chinese with English abstract).

[26] Masondo N A, Kulkarni M G, Finnie J F, Staden J V. Influence of 
biostimulants-seed-priming on, Ceratotheca triloba, germination and seedling growth under low temperatures, low osmotic potential and salinity stress. Ecotox Environ Safe, 2018, 147: 43-48.

[27] Sergio M C, Dante R T, Diodoro G S, Violeta C, Leopoldo M C, Hubert T. Phenotype, seed size and temperature affect the germinative capacity of Agave cupreata. Seed Sci Technol, 2017, 45: 1-6.

[28] 耿广东, 程智慧, 张素勤. 低温发芽鉴定茄子耐冷性的研究. 种子, 2006, 25(6): 43-46.

Geng G D, Cheng Z H, Zhang S Q. Appraisal of eggplant chilling-resistant by low temperature germination. Seed, 2006, 25(6): 43-46 (in Chinese with English abstract).

[29] 王钰静, 谢否, 李志博, 魏亦农, 林海荣. 低温胁迫对北疆棉 花种子萌发的影响及其耐冷性差异评价。种子, 2014, 33(5): $74-77$.

Wang Y J, Xie L, Li Z B, Wei Y N, Lin H R. Effects of low temperature stress to germination of cotton seeds and evaluation of their cold resistance in northern Xinjiang. Seed, 2014, 33(5): 74-77 (in Chinese with English abstract).

[30] 徐建伟, 张晨, 曾晓燕, 张小均, 李志博, 魏亦农. 近十年新 疆北疆主栽棉花种子低温萌发能力差异评价. 新疆农业科学, 2017, 54: 1569-1578.

Xu J W, Zhang C, Zeng X Y, Zhang X J, Li Z B, Wei Y N. Evaluation of seed germination of main-cultivated cotton under low temperature in northern Xinjiang in recent ten years. Xinjiang Agric Sci, 2017, 54: 1569-1578 (in Chinese with English abstract).

[31] 王艺陶, 周宇飞, 李丰先, 依兵, 白薇, 问䑣, 许文娟, 高明超, 黄瑞冬. 基于主成分和 SOM 聚类分析的高粱品种萌发期抗旱 性鉴定与分类. 作物学报, 2014, 40: 110-121.

Wang Y T, Zhou Y F, Li F X, Yi B, Bai W, Yan T, Xu W J, Gao M C, Huang R D. Identification and classification of Sorghum cultivars for drought resistance during germination stage based on principal components analysis and self organizing map cluster analysis. Acta Agron Sin, 2014, 40: 110-121 (in Chinese with English abstract).

[32] 龙卫华, 浦惠明, 张洁夫, 戚存扣, 张学昆. 甘蓝型油菜发芽 期的耐盐性篮选. 中国油料作物学报, 2013, 35: 271-275.

Long W H, Pu H M, Zhang J F, Qi C K, Zhang X K. Screening of Brassica napus for salinity tolerance at germination stage. Chin J Oil Crop Sci, 2013, 35: 271-275 (in Chinese with English abstract).

[33] Tiryaki I, Andrews D J. Germination and seedling cold tolerance in sorghum: I. Evaluation of rapid screening methods. Agron J, 2001, 93: 1386-1391.

[34] Yu J, Tuinstra M R, Claassen M M, Gordon W B, Witt M D. Analysis of cold tolerance in sorghum under controlled environment conditions. Field Crops Res, 2004, 85: 21-30.

\section{附表 13 个主成分的特征值及贡献率}

Supplementary table 1 Eigen value of three principal components and their contributions and cumulative contribution rates

\begin{tabular}{cccc}
\hline $\begin{array}{c}\text { 主成分 } \\
\text { Principal component }\end{array}$ & $\begin{array}{c}\text { 特征值 } \\
\text { Eigen value }\end{array}$ & $\begin{array}{c}\text { 贡献率 } \\
\text { Contribution (\%) }\end{array}$ & $\begin{array}{c}\text { 累计贡献率 } \\
\text { Cumulative contribution (\%) }\end{array}$ \\
\hline I & 2.59 & 43.23 & 43.23 \\
II & 1.71 & 28.56 & 71.79 \\
III & 0.97 & 16.21 & 87.99 \\
\hline
\end{tabular}

附表 2 各因子载荷矩阵

Supplementary table 2 Loading matrix of each component

\begin{tabular}{cccc}
\hline $\begin{array}{c}\text { 萌发指标 } \\
\text { Germination traits }\end{array}$ & $\begin{array}{c}\text { 主成分 I } \\
\text { Principal I }\end{array}$ & $\begin{array}{c}\text { 主成分 II } \\
\text { Principal II }\end{array}$ & $\begin{array}{c}\text { 主成分 III } \\
\text { Principal III }\end{array}$ \\
\hline$X_{1}$ & 0.854 & -0.166 & -0.025 \\
$X_{2}$ & 0.307 & 0.136 & 0.935 \\
$X_{3}$ & 0.272 & 0.897 & 0.030 \\
$X_{4}$ & 0.943 & -0.112 & -0.062 \\
$X_{5}$ & 0.010 & 0.921 & -0.193 \\
$X_{6}$ & 0.899 & -0.053 & -0.237 \\
\hline
\end{tabular}

附表 3 因子得分系数矩阵

Supplementary table 3 Coefficient matrix of component score

\begin{tabular}{cccc}
\hline $\begin{array}{c}\text { 萌发指标 } \\
\text { Germination traits }\end{array}$ & $\begin{array}{c}\text { 主成分 I } \\
\text { Principal I }\end{array}$ & $\begin{array}{c}\text { 主成分 II } \\
\text { Principal II }\end{array}$ & $\begin{array}{c}\text { 主成分 III } \\
\text { Principal III }\end{array}$ \\
\hline$X_{1}$ & 0.329 & -0.097 & -0.026 \\
$X_{2}$ & 0.118 & 0.079 & 0.961 \\
$X_{3}$ & 0.105 & 0.523 & 0.031 \\
$X_{4}$ & 0.363 & -0.065 & -0.064 \\
$X_{5}$ & 0.004 & 0.538 & -0.198 \\
$X_{6}$ & 0.346 & -0.031 & -0.244 \\
\hline
\end{tabular}


附表 4 各品种综合因子得分 $\boldsymbol{Y}$ 值及耐低温特性的排序

Supplementary table $4 \quad Y$-value of comprehensive component score and the ranks of cold-resistance of sorghum hybrids

\begin{tabular}{|c|c|c|c|c|c|}
\hline $\begin{array}{c}\text { 编号 } \\
\text { Serial number }\end{array}$ & $Y_{1}$ & $Y_{2}$ & $Y_{3}$ & $Y$ & $\begin{array}{l}\text { 排序 } \\
\text { Order } \\
\end{array}$ \\
\hline S1 & 0.510 & 0.234 & 0.734 & 0.461 & 16 \\
\hline S2 & 1.058 & 0.156 & 0.403 & 0.644 & 2 \\
\hline S3 & 0.512 & 0.257 & 0.748 & 0.473 & 14 \\
\hline S4 & 1.033 & 0.164 & 0.179 & 0.593 & 6 \\
\hline S5 & 0.282 & 0.169 & 0.385 & 0.264 & 30 \\
\hline S6 & 0.460 & 0.226 & 0.727 & 0.433 & 22 \\
\hline S7 & 0.946 & 0.168 & 0.522 & 0.615 & 4 \\
\hline S8 & 0.921 & 0.141 & 0.532 & 0.596 & 5 \\
\hline S9 & 0.670 & 0.315 & 0.653 & 0.551 & 8 \\
\hline $\mathrm{S} 10$ & 0.473 & 0.215 & 0.619 & 0.416 & 28 \\
\hline S11 & 0.718 & 0.229 & 0.617 & 0.540 & 9 \\
\hline $\mathrm{S} 12$ & 0.531 & 0.264 & 0.686 & 0.473 & 13 \\
\hline S13 & 0.516 & 0.241 & 0.509 & 0.425 & 26 \\
\hline S14 & 0.533 & 0.200 & 0.566 & 0.431 & 23 \\
\hline S15 & 0.511 & 0.255 & 0.636 & 0.451 & 20 \\
\hline S16 & 0.628 & 0.175 & 0.508 & 0.459 & 18 \\
\hline S17 & 0.915 & 0.158 & 0.361 & 0.567 & 7 \\
\hline $\mathrm{S} 18$ & 0.464 & 0.217 & 0.647 & 0.418 & 27 \\
\hline S19 & 0.549 & 0.289 & 0.521 & 0.459 & 17 \\
\hline $\mathrm{S} 20$ & 0.733 & 0.230 & 0.504 & 0.527 & 10 \\
\hline S21 & 0.967 & 0.192 & 0.524 & 0.634 & 3 \\
\hline S22 & 0.386 & 0.211 & 0.762 & 0.398 & 29 \\
\hline S23 & 0.497 & 0.278 & 0.523 & 0.430 & 24 \\
\hline S24 & 0.516 & 0.226 & 0.559 & 0.430 & 25 \\
\hline S25 & 0.560 & 0.225 & 0.595 & 0.458 & 19 \\
\hline S26 & 0.448 & 0.250 & 0.717 & 0.433 & 21 \\
\hline S27 & 0.656 & 0.281 & 0.399 & 0.487 & 12 \\
\hline S28 & 0.512 & 0.246 & 0.710 & 0.462 & 15 \\
\hline S29 & 0.747 & 0.128 & 0.560 & 0.512 & 11 \\
\hline S30 & 1.163 & 0.171 & 0.236 & 0.670 & 1 \\
\hline
\end{tabular}

